\title{
Clinical and Molecular Features of Early Infantile Niemann Pick Type C Disease
}

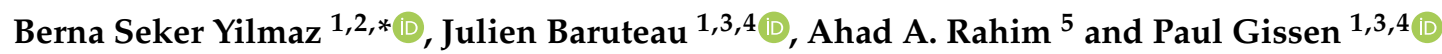 \\ 1 Genetics and Genomic Medicine Department, Great Ormond Street Institute of Child Health, \\ University College London, London WC1N 1EH, UK; j.baruteau@ucl.ac.uk (J.B.); p.gissen@ucl.ac.uk (P.G.) \\ 2 Department of Paediatric Metabolic Medicine, Mersin University, Mersin 33110, Turkey \\ 3 National Institute of Health Research Great Ormond Street Biomedical Research Centre, \\ London WC1N 1EH, UK \\ 4 Metabolic Medicine Department, Great Ormond Street Hospital for Children NHS Foundation Trust, \\ London WC1N 3JH, UK \\ 5 UCL School of Pharmacy, University College London, London WC1N 1AX, UK; a.rahim@ucl.ac.uk \\ * Correspondence: b.yilmaz@ucl.ac.uk
}

Received: 20 June 2020; Accepted: 15 July 2020; Published: 17 July 2020

\begin{abstract}
Niemann Pick disease type C (NPC) is a neurovisceral disorder due to mutations in NPC1 or NPC2. This review focuses on poorly characterized clinical and molecular features of early infantile form of NPC (EIF) and identified 89 cases caused by NPC1 (NPC1) and 16 by NPC2 (NPC2) mutations. Extra-neuronal features were common; visceromegaly reported in 80/89 NPC1 and in 15/16 NPC2, prolonged jaundice in 30/89 NPC1 and 7/16 NPC2. Early lung involvement was present in 12/16 NPC2 cases. Median age of neurological onset was 12 (0-24) and 7.5 (0-24) months in NPC1 and NPC2 groups, respectively. Developmental delay and hypotonia were the commonest first detected neurological symptoms reported in 39/89 and 18/89 NPC1, and in 8/16 and 10/16 NPC2, respectively. Additional neurological symptoms included vertical supranuclear gaze palsy, dysarthria, cataplexy, dysphagia, seizures, dystonia, and spasticity. The following mutations in homozygous state conferred EIF: deletion of exon 1+promoter, c.3578_3591 + 9del, c.385delT, p.C63fsX75, IVS21-2delATGC, c. 2740T >A (p.C914S), c.3584G > T (p.G1195V), c.3478-6T >A, c.960_961dup (p.A321Gfs*16) in NPC1 and c.434T>A (p.V145E), c.199T>C (p.S67P), c.133C>T (p.Q45X), c.141C>A (p.C47X) in NPC2. This comprehensive analysis of the EIF type of NPC will benefit clinical patient management, genetic counselling, and assist design of novel therapy trials.
\end{abstract}

Keywords: Niemann Pick disease type C; early infantile onset; neurological manifestations

\section{Introduction}

Niemann Pick type C (NPC) disease (MIM\#257220 and MIM\#607625) is a progressive, irreversible, and debilitating neurovisceral lysosomal storage disorder characterized by impaired intracellular lipid trafficking, which leads to the accumulation of unesterified cholesterol, sphingosine, and a range of glycosphingolipids in the endolysosomal compartment [1,2].

Bi-allelic mutations in one of the two genes cause NPC: NPC1 (MIM*607623) in 95\% of cases and NPC2 in the remainder (MIM*607625) [1,3,4]. NPC1 (encoded by NPC1) is a large 1278 amino acid transmembrane protein localized to late endosomes and lysosomes. It contains three luminal and 13 transmembrane domains, as well as a lysosomal signal region [5]. The luminal domains are highly glycosylated and have a cholesterol-binding region and a sterol-sensing domain (SSD) [6,7]. A soluble, cholesterol-binding protein, NPC2, is made up of 151 amino acids and four highly conserved domains responsible for cholesterol- binding and release [8-10]. Based on recent studies, the dynamic interface 
between NPC2 and NPC1 proteins facilitates the cholesterol transport by reducing the energy barrier and stabilizing the passage [11].

The estimated incidence of this devastating disease is approximately $1 / 100,000$ births, with variations between ethnic groups [12]. Higher incidence has been reported in some isolated populations such as Acadians in Nova Scotia, Hispanics from Southern Colorado and New Mexico, and Greeks on a small Aegean island due to a founder effect [1,13-15]. It is also suggested that the adult-onset form may have a higher prevalence at 1/19,000-36,000 [16]

The clinical spectrum and progression of disease are extremely heterogeneous [17,18]. Disease onset occurs across the lifespan, from the prenatal period to adulthood and a range of visceral, neurological, and psychiatric clinical features seem to appear and progress differently in individual patients $[1,3,12,17,19,20]$. The age of onset of neurological symptoms determines the speed of progression of the disease and allows life expectancy predictions to be made [1]. Hence, NPC is best classified according to the age of onset of neurological manifestations as follows:

1. Visceral-neurodegenerative form

- $\quad$ Early-infantile (neurological onset $<2$ years)

2. Neurodegenerative form

- $\quad$ Late-infantile (neurological onset 2-6 years)

- Juvenile (neurological onset 6-15 years)

3. Psychiatric-neurodegenerative form

- $\quad$ Adult (neurological onset $>15$ years) [12]

More than $40 \%$ of patients with all forms of NPC present in the first year of life with cholestatic jaundice and visceromegaly, usually hepatosplenomegaly $[1,17]$. Although jaundice and hepatomegaly disappear after 6-12 months in most patients, splenomegaly usually remains [20,21]. Very infrequently (probably $<5 \%$ of the total NPC population) patients present in the first 2 weeks of life with liver failure leading to death unless liver transplantation is performed [22]. In such rare cases patients may recover after liver transplant and have a variable period of reasonable health before succumbing to neurological disease [23-26]. Moreover, there is another distinct phenotype, known as fetal onset NPC which presents with fetal ascites/nonimmune hydrops fetalis [1,12,27-29].

In the majority of patients, neurological symptoms start insidiously after a varying period of normal or slightly delayed development. While initial symptoms are often nonspecific such as hypotonia, developmental delay, and clumsiness, regression gradually occurs with patients experiencing loss of motor skills and cognitive decline $[1,17,30]$. Vertical supranuclear gaze palsy, cataplexy, or drop attacks could occur early in the presentation of NPC. Ataxia, dysphagia, dysarthria, and loss of cognitive skills demonstrate progression of the disease and psychiatric symptoms are typical in adult patients [4,31-33]. Patients with early onset NPC have a more severe and rapidly progressing course than those with later onset of neurological disease $[1,12,27,34,35]$.

Whilst the features and rate of progression of the common juvenile form of NPC are well known, the specific disease characteristics of the rarer early infantile form (EIF) is not well established. The dearth of knowledge in this area impairs provision of accurate prognosis for the families and potentially inhibits design of clinical trials for novel therapies. Thus, in this article we aimed to provide better insight into this form of the disease on the basis of our review of published cases.

\section{Results}

\subsection{Literature Search}

Keyword “Niemann Pick Disease type C" yielded 1097, keyword “Niemann Pick Disease” gave 3638 publications. A total of 181 articles underwent full text review. According to the reference list 
check 22 additional articles were assessed and in 28 of publications patients met our inclusion criteria. Articles included in this study were published between 1988 and 2020. Figure 1 shows a flowchart of the search process for the publications included in this study.

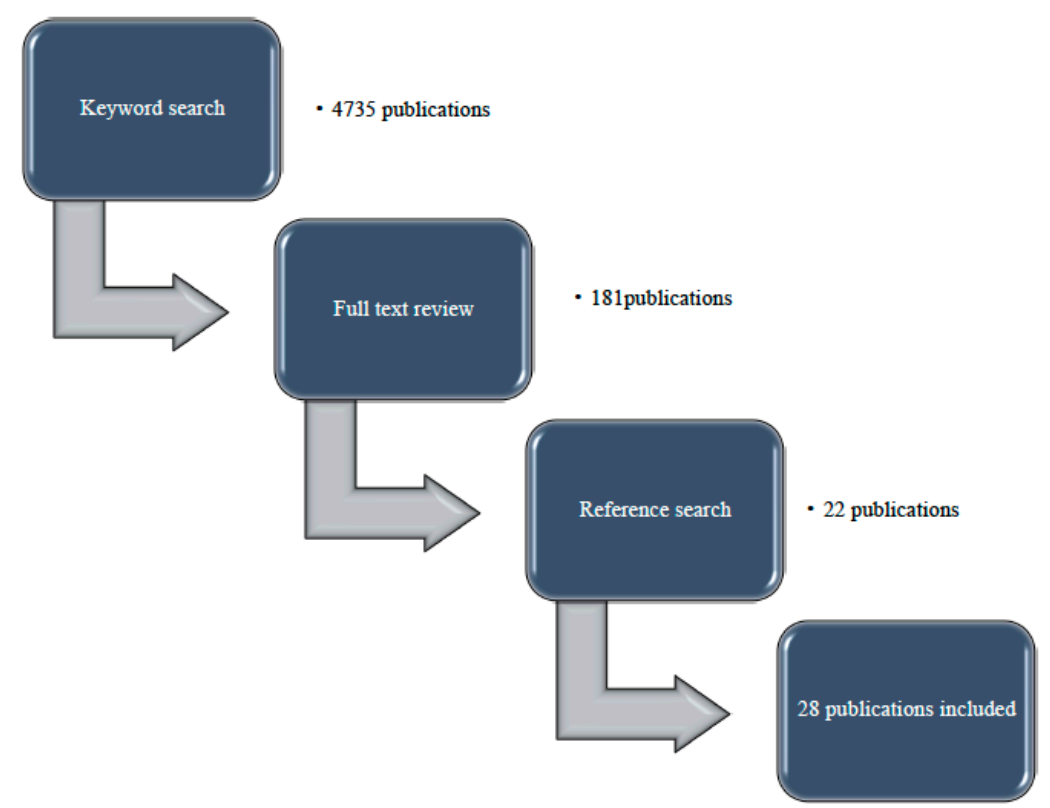

Figure 1. Flowchart of the selection process for the publications included.

\subsection{Early Infantile Cases with NPC1 Mutations}

Several international publications reported clinical and molecular findings in their cohorts of NPC cases (Table 1). In the Czech Republic, the estimated prevalence of NPC was suggested as 0.93 per 100,000 births [36] and 56 NPC patients, 30 (54\%) females and 26 (46\%) males, were reported in an observational, retrospective analysis of all NPC cases, diagnosed between 1975 and 2012. A total of 21 patients (38\%) from 10 families were relatives; siblings and cousins. A total of 55 patients were diagnosed with NPC1 and one was diagnosed with NPC2. A total of 8 of 55 NPC1 patients $(14.5 \%)$ had EIF (six NPC1, 1 NPC2 mutations and two with unknown genetics) [36]. Psychomotor retardation or regression between 6 months and 2 years of age were the most common symptoms among EIF patients. All of the EIF patients had visceral symptoms including neonatal hepatosplenomegaly and/or prolonged neonatal jaundice. Ataxia and speech retardation occurred around 2 years of age and was followed by gelastic cataplexy, seizures, oculomotor abnormalities, dysphagia, and spasticity. Most of these patients deceased around 5 years of age due to respiratory complications [36].

Table 1. Summary of the Niemann Pick disease type C (NPC) studies.

\begin{tabular}{ccccccc}
\hline Reference & Country & Methodology & $\begin{array}{c}\text { Total Number } \\
\text { of Patients } \\
\text { NPC1/NPC2 }\end{array}$ & $\begin{array}{c}\text { Number of } \\
\text { EIF Patients } \\
\text { NPC1/NPC2 }\end{array}$ & $\begin{array}{c}\text { Most Common } \\
\text { Neurological } \\
\text { Symptom among EIF } \\
\text { Patients }\end{array}$ & $\begin{array}{c}\text { Median Age } \\
\text { of Death } \\
\text { (Months) }\end{array}$ \\
\hline$[36]$ & $\begin{array}{c}\text { Czech } \\
\text { Republic }\end{array}$ & $\begin{array}{c}\text { Observational } \\
\text { retrospective }\end{array}$ & $55 / 1$ & $6 / 1$ & $\begin{array}{c}\text { Psychomotor } \\
\text { Retardation/regression }\end{array}$ & 60 \\
\hline$[37]$ & UK & $\begin{array}{c}\text { Observational } \\
\text { retrospective }\end{array}$ & $110 / 2$ & $8 / 0$ & DD, ataxia, dysarthria & 65 \\
\hline$[2]$ & International & $\begin{array}{c}\text { Observational } \\
\text { prospective }\end{array}$ & $134 / 3$ & 16 & DD, dysphagia and \\
VSGP & \\
\hline$[38]$ & Spain & $\begin{array}{c}\text { Mutation } \\
\text { screening }\end{array}$ & $40 / 0$ & 12 & & \\
\hline$[39]$ & Spain & $\begin{array}{c}\text { Mutation } \\
\text { screening }\end{array}$ & $30 / 0$ & 10 & & \\
\hline
\end{tabular}


Table 1. Cont.

\begin{tabular}{ccccccc}
\hline Reference & Country & Methodology & $\begin{array}{c}\text { Total Number } \\
\text { of Patients } \\
\text { NPC1/NPC2 }\end{array}$ & $\begin{array}{c}\text { Number of } \\
\text { EIF Patients } \\
\text { NPC1/NPC2 }\end{array}$ & $\begin{array}{c}\text { Most Common } \\
\text { Neurological } \\
\text { Symptom among EIF } \\
\text { Patients }\end{array}$ & $\begin{array}{c}\text { Median Age } \\
\text { of Death } \\
\text { (Months) }\end{array}$ \\
\hline$[40]$ & Italy & $\begin{array}{c}\text { Mutation } \\
\text { screening }\end{array}$ & $32 / 2$ & $11 / 2$ & Hypotonia, ataxia & Hypotonia, DD \\
\hline$[41]$ & Italy & $\begin{array}{c}\text { Mutation } \\
\text { screening }\end{array}$ & $97 / 8$ & $21 / 3$ & \\
\hline$[42]$ & France & $\begin{array}{c}\text { Prospective } \\
\text { open-label }\end{array}$ & $19 / 1$ & $8 / 0$ & DD \\
\hline$[44]$ & Egypt & $\begin{array}{c}\text { Observational } \\
\text { descriptive }\end{array}$ & $23 / 0$ & $6 / 0$ & \\
\hline$[45]$ & Iran & $\begin{array}{c}\text { Observational } \\
\text { descriptive }\end{array}$ & $21 / 0$ & $3 / 0$ & Frequent falls, DD \\
\hline$[46]$ & Iran & $\begin{array}{c}\text { Observational } \\
\text { case series }\end{array}$ & $11 / 0$ & $5 / 0$ & \\
\hline$[21]$ & $\begin{array}{c}\text { Germany } \\
\text { Switzerland }\end{array}$ & $\begin{array}{c}\text { Biochemical/genetic } \\
\text { screening }\end{array}$ & $11 / 1$ & $6 / 1$ & \\
\hline$[47]$ & Israel & ansectional & $41 / 1$ & $5 / 1$ & \\
\hline
\end{tabular}

The prevalence of NPC in the UK is estimated as 0.78 per 100,000 birth [37]. An observational retrospective study revealed a total of 146 NPC patients born between 1954 and 2009, among whom $77(53 \%)$ were female and $69(47 \%)$ male [37]. A total of 112 patients $(77 \%)$ had at least one identified disease-causing mutation; 110 had NPC1 mutations and two had NPC2 mutations. Six patients (4\%) had the visceral neonatal form, eight patients (5\%) had EIF, 51 patients (35\%) had LIF, $42(29 \%)$ had juvenile and $25(17 \%)$ had adolescent/adult phenotype [37]. The mean (SD; range) age at onset of neurological manifestations in EIF group was $1.1(0.7 ; 0-2.0)$ years. Moreover, the mean (SD) time between onset of neurological manifestations and diagnosis was 0.26 (1.49) years, with diagnostic testing based on detection of visceral symptoms in four patients. The median age at death was 65 months, ranged 40-101 months in this group which was reported as death related to NPC. A total of 6/8 patients (75\%) exhibited both prolonged neonatal jaundice and hepatosplenomegaly. Most frequent neurological symptoms were developmental delay, ataxia, and dysarthria which were developed in all eight patients. Cataplexy/epileptic seizures and swallowing difficulties were each recorded in 6/8 (75\%) patients. Ophthalmic assessments revealed vertical supranuclear gaze palsy (VSGP) in 5/8 (63\%) of EIF patients [37].

The International NPC registry, a prospective observational cohort study, included 163 NPC patients from 14 European countries, Australia, Brazil, and Canada and 137 patients had available genetic results; 134 had NPC1 and three had NPC2 mutations. A total of 16 patients (11\%) were in EIF group [2]. Almost all EIF patients had visceromegaly and/or prolonged jaundice and eight out of $16(50 \%)$ were diagnosed before the appearance of neurological manifestations. Three patients in the EIF onset group presented with perinatal hypotonia. Developmental delay, dysphagia, and VSGP were the most common neurological manifestations among the EIF cases [2].

A total of 40 Spanish NPC1 patients diagnosed between 1988 and 2003 were presented in one study [34] where the only available clinical data was the presence of visceromegaly and neonatal jaundice. Though the publication lacked expanded neurological information, as many as $12 / 40(30 \%)$ patients were determined as "severe infantile" with a neurological presentation in the first 2 years of life [38].

Similar to the above study, 30 NPC1 patients who were referred for molecular analysis from Spain were described in another report. According to the age of neurological onset, three (10\%) neonatal, $10(33.3 \%)$ EIF, six (20\%) LIF, six (20\%) juvenile, and two (6.6\%) adult cases were noted [39]. 
In a multicenter study from Italy, 44 NPC patients were reported. A total of 41/44 patients had NPC1 mutations and 11/41 (26.8\%) were in the EIF group [40]. A total of three NPC1 cases who died during the first month of life due to liver or respiratory insufficiency without signs of neurological involvement were labelled as early infantile systemic lethal form (EISL) [40].

In a more recent updated publication, 105 patients from 83 unrelated families were included in a collaborative multicenter study aimed at characterizing the molecular bases of Niemann-Pick $C$ in Italy [41]. Clinical phenotypes were classified according to the age at onset of neurological symptoms and 97/105 cases had NPC1 mutations and $21.9 \%$ of these NPC1 cases were EIF patients [41].

A prospective, open-label study reported all pediatric NPC patients treated with miglustat in France between October 2006 and December 2010, adult cases were not included. A total of 19/20 cases were NPC1 patients, and 8/19 (42\%) had EIF presentation [42]. A history of hepatosplenomegaly and/or neonatal jaundice was recorded in all eight EIF patients and liver biopsy revealed evidence of cirrhosis in two patients [42]. In EIF group, neurological symptoms including hypotonia, developmental delay and swallowing difficulties were first detected between 5 and 12 months of age [42]. VSGP was observed at 9,18 , and 24 months of age in five patients, but no patients had cataplexy [42]. One patient had significant dysphagia at 5 months of age, requiring enteral feeding with nasogastric tube, gastrostomy was inserted at the age of 9 months [42]. Four patients had peripheral neuropathy and distal motor deficit [42].

A total of 23 NPC1 patients were reported from Egypt in a study designed to describe the spectrum of clinical, biochemical and molecular profile of the disease [43]. Disease onset was reported as neonatal in eight patients (presenting $<3$ months of age), EIF in six (presentation from 3 months-2 years of age), LIF in three (2-6 years), and juvenile in six patients (6-15 years), nevertheless, adult patients were not reported in this cohort [43]. Nineteen patients were offspring of consanguineous marriages $(82.6 \%)$, while positive family history was reported in 13 families (65\%) [43]. Age of neurological onset varied between 8 and 18 months in the EIF group while all of these cases had also visceral symptoms [43].

A total of 21 NPC patients diagnosed between 2009 and 2012 were included in an observational study from Iran [41]. All patients were from consanguineous parents and three of them had EIF. Among EIF and LIF patients, $70 \%$ had hepatomegaly with or without spleen involvement and $40 \%$ had prolonged neonatal jaundice as a presenting feature. Neurodevelopmental delay was seen in all three patients with EIF [44]. In another Iranian cohort, 11 NPC patients were reported. A total of 5/11 were EIF patients with accompanying visceromegaly and three of them died in the first 2 years of life [45].

A publication from China reported that 7/12 total NPC cohort had EIF disease [42]. A total of 6/7 had NPC1 mutations, 4/6 had splenomegaly, and 2/6 had hepatosplenomegaly. Frequent falls and developmental delay were the most common neurological symptoms [46].

A cross-sectional analysis of 42 NPC patients residing in Germany or Switzerland were designed to assess neuropsychiatric symptoms. A total of $6 / 42$ patients had a neurological onset in the first 2 years of life, five of whom had NPC1 mutations [21].

A retrospective study from Israel reported 12 patients from six nuclear families of Bedouin origin. A total of 5/12 patients had EIF with NPC1 mutations and all of them died before 5 years of age [47].

Overall, we identified 89 cases reported as EIF NPC due to NPC1 mutations (Table 2). There were $40(45 \%)$ females and $23(26 \%)$ males and $26(29 \%)$ of unknown gender. While the exact age of neurological symptom onset was included in 66 cases, 23 cases were only determined as "early infantile Niemann Pick type C". In these 66 cases, median age of neurological onset was 12 months (range 0-24 months). Positive family history was noted in 27 patients and there were five pairs of siblings. Consanguinity status was mentioned in 35 cases and 30 of them were from consanguineous parents.

As expected, visceral symptoms were very frequent among the EIF NPC patients (Figure 2). The presence of visceromegaly has been noted in $80 / 89$ cases (89.9\%). A total of $54 / 89$ were determined as hepatosplenomegaly (HSM), 11/89 as splenomegaly (SM), and 15/89 as visceromegaly (VM). In four cases, there was no visceromegaly and status of visceromegaly was unknown in five cases. Prolonged jaundice was reported in 30/89 cases. 
Table 2. Demographic, clinical, and molecular features of early infantile neurological onset disease with NPC1 mutations.

\begin{tabular}{|c|c|c|c|c|c|c|c|c|}
\hline $\begin{array}{l}\text { Patient } \\
\text { No }\end{array}$ & $\begin{array}{l}\text { Initial Neurological } \\
\text { Symptom/s (Age First } \\
\text { Reported in Months) }\end{array}$ & $\begin{array}{l}\text { Visceral } \\
\text { Symptoms }\end{array}$ & Sex & Ethnicity & Allele 1 & Allele 2 & $\begin{array}{l}\text { Age of } \\
\text { Death } \\
\text { (Months) }\end{array}$ & Ref. \\
\hline 1 & $\begin{array}{l}\text { Swallowing difficulties lack of } \\
\text { motor coordination (3) }\end{array}$ & HSM, PJ & $\mathrm{F}$ & Spanish & del promotor+exon 1 & del promotor+exon 1 & NK & [48] \\
\hline 2 & $\begin{array}{l}\text { Severe encephalopathy with } \\
\text { uncontrolled movements, } \\
\text { ataxia, tremor, nystagmus ( } 24)\end{array}$ & SM & M & Spanish & c.385delT & c.385delT & NK & [48] \\
\hline 3 & NK & HSM, PJ & $\mathrm{F}$ & Spanish & c.2830G>A (p.D944N) & c.3104C>T (p.A1035V) & NK & [48] \\
\hline 4 & NK (22) & HSM & $\mathrm{M}$ & Spanish & c.1757delA & c.2746_2748delAAT & NK & [48] \\
\hline 5 & Hypotonia (5) & HSM, PJ & $\mathrm{F}$ & French & c.1138C > T (p.L380F) & c.2872C>T (p.R958X) & NK & [42] \\
\hline 6 & Hypotonia, DD (6) & HSM, PJ & $\mathrm{F}$ & French & p.C63fsX75 & p.C63fsX75 & NK & {$[42]$} \\
\hline 7 & Hypotonia, DD (7) & HSM & $\mathrm{F}$ & French & c.3614C >G (p.T1205R) & c.3614C >A (p.T1205K) & NK & [42] \\
\hline 8 & Hypotonia, DD (9) & HSM, PJ & $\mathrm{F}$ & French & IVS21-2del ATGC & IVS21-2del ATGC & 33 & [42] \\
\hline 9 & Hypotonia, DD (9) & HSM, PJ & $\mathrm{F}$ & French & c.3584G>T (p.G1195V) & c.3584G>T (p.G1195V) & NK & [42] \\
\hline 10 & Hypotonia, DD (10) & HSM, PJ & $\mathrm{F}$ & French & c. $1298 \mathrm{C}>\mathrm{T}$ (p.P433L) & IVS14+1G>A & NK & [42] \\
\hline 11 & Hypotonia, DD (12) & HSM, PJ & $\mathrm{F}$ & French & c. $1298 \mathrm{C}>\mathrm{T}$ (p.P433L) & p.T1205fs & NK & {$[42]$} \\
\hline 12 & Hypotonia, DD (12) & HSM, PJ & F & French & c.3107C $>$ T (p.T1036M) & c.3107C > T (p.T1036M) & NK & [42] \\
\hline 13 & Frequent falls, DD (12) & SM & $\mathrm{F}$ & Chinese & c.1501G>T (p.D501Y) & c.1800delC (P.I601FfsX13) & NK & [46] \\
\hline 14 & Frequent falls, DD (18) & SM & $\mathrm{M}$ & Chinese & c.416dupC (p.N140KfsX30) & c.1832A>G (p.D611G) & NK & {$[46]$} \\
\hline 15 & Frequent falls, DD (12) & HSM, PJ & $\mathrm{M}$ & Chinese & c.2177G >C (p.R726T) & c.3734_3735delCT (p.P1245RfsX12) & NK & [46] \\
\hline 16 & $\mathrm{DD}(20)$ & HSM & $\mathrm{M}$ & Chinese & c.2230_2231delGT (p.V744SfsX27) & c.3734_3735delCT (p.P1245RfsX12) & NK & {$[46]$} \\
\hline 17 & Frequent falls (15) & SM & $\mathrm{F}$ & Chinese & c.1553G>A (p.R518Q) & c.2795dupA & NK & [46] \\
\hline 18 & Frequent falls (20) & SM & $\mathrm{M}$ & Chinese & c.1553G>A (p.R518Q) & c.2795dupA & NK & [46] \\
\hline 19 & $\mathrm{DD}(\mathrm{NK})$ & No & $\mathrm{M}$ & UK & c.2819C > T (p.S940L) & NK & 92 & [37] \\
\hline 20 & DD (NK) & VM, PJ & $\mathrm{F}$ & UK & c.3557G>A (p.R1186H) & c.3107C > T (p.T1036M) & 101 & [37] \\
\hline 21 & $\mathrm{DD}(\mathrm{NK})$ & VM, PJ & $\mathrm{F}$ & UK & c.3557G >A (p.R1186H) & c.3107C>T (p.T1036M) & 85 & [37] \\
\hline 22 & DD (17) & No & $\mathrm{F}$ & UK & c.3503G >A (p.C1168Y) & c.3503G>A (p.C1168Y) & 77 & [37] \\
\hline 23 & $\mathrm{DD}(\mathrm{NK})$ & PJ, VM & $\mathrm{F}$ & UK & c.3578_3591 + 9del & c.3578_3591 + 9del & 40 & [37] \\
\hline 24 & $\mathrm{DD}(\mathrm{NK})$ & PJ, VM & $\mathrm{F}$ & UK & c.2801G>A (p.R934Q) & c.2978del (p.G993EfsX4) & 53 & [37] \\
\hline 25 & $\mathrm{DD}(12)$ & HSM, PJ & $\mathrm{M}$ & Czech & c.3557G>A (p.R1186H) & NK & 60 & [36] \\
\hline 26 & DD (12) & HSM & $\mathrm{F}$ & Czech & c.3182T>C (p.I1061T) & c. $3591+1 \mathrm{G}>\mathrm{A}$ & 132 & [36] \\
\hline
\end{tabular}


Table 2. Cont

\begin{tabular}{|c|c|c|c|c|c|c|c|c|}
\hline $\begin{array}{l}\text { Patient } \\
\text { No }\end{array}$ & $\begin{array}{l}\text { Initial Neurological } \\
\text { Symptom/s (Age First } \\
\text { Reported in Months) }\end{array}$ & $\begin{array}{l}\text { Visceral } \\
\text { Symptoms }\end{array}$ & Sex & Ethnicity & Allele 1 & Allele 2 & $\begin{array}{c}\text { Age of } \\
\text { Death } \\
\text { (Months) }\end{array}$ & Ref. \\
\hline 27 & DR (20) & HSM & $\mathrm{F}$ & Czech & c.1812dupT (p.Ala605Cysfs²) & c.3558delC (p.A1187Rfs*54) & 48 & [36] \\
\hline 28 & Speech retardation (22) & HSM, PJ & $\mathrm{F}$ & Czech & c.3557G >A (p.R1186H) & c.826T >C (p.Y276H) & NK & [36] \\
\hline 29 & DR, ataxia (18) & HSM & $\mathrm{F}$ & Czech & c.3557G >A (p.R1186H) & c.2196dupT (p.Pro733Serfs*10) & NK & [36] \\
\hline 30 & $\mathrm{DD}$, ataxia (22) & SM & $\mathrm{F}$ & Czech & c.3557G $>$ A (p.R1186H) & c.3614C >A (p.T1205K) & 72 & [36] \\
\hline 31 & $\mathrm{DD}(12)$ & HSM & $\mathrm{F}$ & Spanish & c.319delc & Nucleotide +5 at intron 18 & NK & [49] \\
\hline 32 & Speech regression (18) & SM & $\mathrm{F}$ & Japanese & c.2108T >C (p.F703S) & c. $2438 \mathrm{C}>\mathrm{G}(\mathrm{p} . \mathrm{S} 813 \mathrm{X})$ & 108 & {$[50]$} \\
\hline 33 & $\begin{array}{l}\text { Hypotonia (2), } \\
\text { DD (4) }\end{array}$ & HSM, PJ & $\mathrm{M}$ & Japanese & c. $2783 \mathrm{~A}>\mathrm{C}$ (p.Q928P) & c.3008T $>$ G (p.L1003R) & NK & [51] \\
\hline 34 & Hypotonia (1) & HSM & NK & Spanish & c.2826G >T (p.W942C) & c.2883_2897del15 (p.Ile962_Phe966del) & NK & [38] \\
\hline 35 & Hypotonia, DD (12) & HSM & NK & Spanish & c.3104C > T (p.A1035V) & c.3104C $>$ T (p.A1035V) & NK & [38] \\
\hline 36 & Hypotonia (Newborn) & HSM & NK & Spanish & c.530G >A (p.C177Y) & c.2876T>A (p.V959E) & NK & [38] \\
\hline 37 & $\begin{array}{l}\text { Motor regression, spastic } \\
\text { tetraparesis (12) }\end{array}$ & No & NK & Spanish & c. $955+1 \mathrm{G}>\mathrm{A}(\mathrm{IVS} 7+5 \mathrm{G}>\mathrm{A})$ & c.2826G > T (p.W942C) & NK & [38] \\
\hline 38 & Nystagmus (8) & HSM & NK & Spanish & c.1935T>A (p.C645X) & c.3236T>C (p.F1079S) & NK & [38] \\
\hline 39 & $\mathrm{DD}(3)$ & HSM, PJ & $\mathrm{M}$ & Iranian & c.2925_2928delCTGC (p.C976fs) & c.2925_2928delCTGC (p.C976fs) & NK & [44] \\
\hline 40 & NK (8) & VM & $\mathrm{F}$ & Egyptian & c.3380dupT (p.M1127Ilfs*131) & c.3380dupT (p.M1127Ifs*131) & NK & [43] \\
\hline 41 & NK (4) & $\mathrm{VM}$ & $\mathrm{M}$ & Egyptian & c.425_426delAA (p.K142Rfs*27) & c.425_426delAA (p.K142Rfs*27) & NK & [43] \\
\hline 42 & NK (6) & $\mathrm{VM}$ & $\mathrm{M}$ & Egyptian & c. $2872 C>T$ (p.R958X) & c. $2872 C>T$ (p.R958X) & NK & [43] \\
\hline 43 & NK (4) & VM & $\mathrm{M}$ & Egyptian & c. $2245+1 \mathrm{G}>\mathrm{A}$ & c. $2245+1 G>A$ & NK & [43] \\
\hline 44 & NK (9) & $\mathrm{VM}$ & $\mathrm{M}$ & Egyptian & c.2972_2973delAG (p.Q991Rfs) & c.2972_2973delAG (p.Q991Rfs) & NK & [43] \\
\hline 45 & NK (10) & VM & $\mathrm{F}$ & Egyptian & c.2972_2973delAG (p.Q991Rfs) & c.2972_2973delAG (p.Q991Rfs) & NK & [43] \\
\hline 46 & NK $(4)$ & $\mathrm{VM}$ & M & Egyptian & $\begin{array}{l}\text { Duplication/multiple copies of } \\
\text { exons } 10 \text { and } 11\end{array}$ & $\begin{array}{l}\text { Duplication/multiple copies of exons } \\
\qquad 10 \text { and } 11\end{array}$ & NK & [43] \\
\hline 47 & NK (8) & $\mathrm{VM}$ & $\mathrm{F}$ & Egyptian & c.3032_3038delins10bp (p.C1011*) & c.3032_3038delins10bp (p.C1011*) & NK & [43] \\
\hline 48 & NK (18) & $\mathrm{VM}$ & $\mathrm{F}$ & Egyptian & c.2972_2973delAG (p.Q991Rfs) & c.2972_2973delAG (p.Q991Rfs) & NK & [43] \\
\hline 49 & Hypotonia (24) & HSM & $\mathrm{F}$ & Portuguese & IVS23+1G $>$ A & IVS23+1G>A & NK & [52] \\
\hline 50 & $\mathrm{DD}(12)$ & HSM & NK & Portuguese & c.3104C>T (p.A1035V) & c.3104C $>$ T (p.A1035V) & 54 & [52] \\
\hline 51 & Hypotonia (23) & HSM, PJ & NK & Portuguese & IVS23+1G $>$ A & c.3104C $>$ T (p.A1035V) & 36 & [52] \\
\hline 52 & NK (18) & $\mathrm{VM}$ & $\mathrm{M}$ & Egyptian & c. 2872 C > T (p.R958X) & c. $2872 \mathrm{C}>\mathrm{T}$ (p.R958X) & NK & [43] \\
\hline
\end{tabular}


Table 2. Cont.

\begin{tabular}{|c|c|c|c|c|c|c|c|c|}
\hline $\begin{array}{l}\text { Patient } \\
\text { No }\end{array}$ & $\begin{array}{l}\text { Initial Neurological } \\
\text { Symptom/s (Age First } \\
\text { Reported in Months) }\end{array}$ & $\begin{array}{c}\text { Visceral } \\
\text { Symptoms }\end{array}$ & Sex & Ethnicity & Allele 1 & Allele 2 & $\begin{array}{c}\text { Age of } \\
\text { Death } \\
\text { (Months) }\end{array}$ & Ref. \\
\hline 53 & NK (11) & $\mathrm{VM}$ & $\mathrm{M}$ & Egyptian & c.451_452delAG (p.S151Ffs*18) & c.451_452delAG (p.S151Ffs*18) & NK & [43] \\
\hline 54 & DR (NK) & HSM & $\mathrm{F}$ & Iranian & c.2920_2923delCCTG (p.C976Ffs*6) & c.2920_2923delCCTG (p.C976Ffs*6) & 24 & [45] \\
\hline 55 & DR (NK) & HSM & $\mathrm{M}$ & Iranian & c. $2740 \mathrm{~T}>\mathrm{A}$ (p.C914S) & c. $2740 \mathrm{~T}>\mathrm{A}$ (p.C914S) & NK & [45] \\
\hline 56 & DR (NK) & HSM & $\mathrm{F}$ & Iranian & c.1415T>C (p.L472P) & c.1415T>C (p.L472P) & NK & [45] \\
\hline 57 & $\mathrm{DR}(\mathrm{NK})$ & HSM & $\mathrm{F}$ & Iranian & c.3478-6T>A & c.3478-6T>A & 28 & [45] \\
\hline 58 & DR (NK) & HSM & $\mathrm{F}$ & Iranian & c.960_961dup (p.A321Gfs*16) & c.960_961dup (p.A321Gfs*16) & 7 & [45] \\
\hline 59 & DD, Ataxia (24) & HSM & $\mathrm{F}$ & Greek & c.3394G >A (p.A1132P) & c.3394G >A (p.A1132P) & NK & [13] \\
\hline 60 & NK & HSM & NK & Italian & p.F284LfsX26 & p.F284LfsX26 & NK & {$[40]$} \\
\hline 61 & NK & SM & NK & Italian & c.2972_2973delAG (p.Q991Rfs) & c.2972_2973delAG (p.Q991Rfs) & NK & [40] \\
\hline 62 & NK & HSM & NK & Italian & c.1819C>T (p.R607X) & c.3614C>A (p.T1205K) & 36 & {$[40]$} \\
\hline 63 & NK & SM & NK & Italian & c.93_94delTG (p.C31WfsX26) & c.93_94delTG (p.C31WfsX26) & NK & [40] \\
\hline 64 & NK & HSM & NK & Italian & c. $464-2 \mathrm{~A}>\mathrm{C}$ & c. $464-2 \mathrm{~A}>\mathrm{C}$ & NK & [40] \\
\hline 65 & NK & HSM & NK & Italian & c. $464-2 \mathrm{~A}>\mathrm{C}$ & c. $464-2 \mathrm{~A}>\mathrm{C}$ & NK & {$[40]$} \\
\hline 66 & NK & HSM & NK & Italian & c.3467A>G (p.N1156S) & c.3467A>G (p.N1156S) & NK & [40] \\
\hline 67 & NK & HSM & NK & Italian & c.3613dupA (p.T1205NfsX53) & c.3613dupA (p.T1205NfsX53) & NK & {$[40]$} \\
\hline 68 & NK & HSM & NK & Italian & c.2800C>T (p.R934X) & c.2872C>T (p.R958X) & 36 & [40] \\
\hline 69 & NK & HSM & NK & Italian & c.2829C >G (p.I943M) & NK & NK & [40] \\
\hline 70 & NK & NK & $\mathrm{F}$ & German & c.2071C>T (p.P691S) & c.2279_2281TCTdel (p.Phe760del) & NK & [21] \\
\hline 71 & NK (12) & NK & $\mathrm{F}$ & German & $\begin{array}{l}\text { c.352_353delAG } \\
\text { p.(Gln119Valfs*8) }\end{array}$ & $\begin{array}{l}\text { c.352_353delAG } \\
\text { p.(Gln119Valfs*8) }\end{array}$ & 72 & [21] \\
\hline 72 & NK (24) & NK & $\mathrm{F}$ & German & c.3047A>T (p.H1016L) & c.3182T>C (p.I1061T) & NK & [21] \\
\hline 73 & NK (24) & NK & $\mathrm{F}$ & German & p.S940L & NK & NK & [21] \\
\hline 74 & NK (24) & NK & $\mathrm{M}$ & German & c.3019C>G (p.P1007A) & c.2873G>A (p.R958Q) & NK & [21] \\
\hline 75 & $\mathrm{DD}(5)$ & SM, PJ & $\mathrm{M}$ & Iranian & c.1415T>C (p.L472P) & c.1415T>C (p.L472P) & NK & [53] \\
\hline 76 & DD, DR (9) & SM & $\mathrm{F}$ & Iranian & c.1415T>C (p.L472P) & c.1415T>C (p.L472P) & NK & [53] \\
\hline 77 & Hypotonia, DD (NK) & PJ & M & Greek & $\begin{array}{c}\text { IVS23 + 3insT } \\
\text { (c.3591 + 3insT) }\end{array}$ & NK & 42 & [54] \\
\hline 78 & Hypotonia, DD, Dystonia (5) & HSM, PJ & $\mathrm{F}$ & Greek & c.852delT (p.F284Lfs*26) & del promotor+exon1-10 & 26 & [54] \\
\hline
\end{tabular}


Table 2. Cont

\begin{tabular}{|c|c|c|c|c|c|c|c|c|}
\hline $\begin{array}{l}\text { Patient } \\
\text { No }\end{array}$ & $\begin{array}{l}\text { Initial Neurological } \\
\text { Symptom/s (Age First } \\
\text { Reported in Months) }\end{array}$ & $\begin{array}{l}\text { Visceral } \\
\text { Symptoms }\end{array}$ & Sex & Ethnicity & Allele 1 & Allele 2 & $\begin{array}{l}\text { Age of } \\
\text { Death } \\
\text { (Months) }\end{array}$ & Ref. \\
\hline 79 & Hypotonia (3) & PJ, HSM & $\mathrm{M}$ & Greek & c.275A>G (p.Q92R) & c.3557T>C (p.C119*) & NK & {$[54]$} \\
\hline 80 & $\mathrm{DD}(9)$ & PJ, HSM & M & Greek & c.3265G>A (p.E1089K) & c.2102-2103insA (p.N701Kfs*13) & NK & {$[54]$} \\
\hline 81 & $\mathrm{DD}(3)$ & PJ, HSM & NK & Bedouin-Israeli & c.1211G >A (p.R404Q) & c.1211G > A (p.R404Q) & 40 & {$[47]$} \\
\hline 82 & DD, DR (12) & PJ, HSM & NK & Bedouin-Israeli & c.1211G >A (p.R404Q) & c.1211G > A (p.R404Q) & 35 & {$[47]$} \\
\hline 83 & DD, DR (24) & HSM & NK & Bedouin-Israeli & c.1211G >A (p.R404Q) & c.1211G > A (p.R404Q) & 53 & {$[47]$} \\
\hline 84 & DD, DR (24) & HSM, PJ & NK & Bedouin-Israeli & c.1211G >A (p.R404Q) & c.1211G > A (p.R404Q) & 46 & [47] \\
\hline 85 & DD, DR (18) & HSM, PJ & NK & Bedouin-Israeli & c.1211G >A (p.R404Q) & c.1211G > A (p.R404Q) & 52 & [47] \\
\hline 86 & Hypotonia, abnormal gait (20) & HSM & NK & French & c.1211G >A (p.R404Q) & c.709C $>$ T (p.237S) & 64 & {$[55,56]$} \\
\hline 87 & $\mathrm{DD}(12)$ & HSM, PJ & NK & French & c.2324A>C (p.Q775P) & c.2324A >C (p.Q775P) & 44 & {$[55,56]$} \\
\hline 88 & $\mathrm{DD}(10-12)$ & HSM, PJ & NK & French & c.1892T>G (p.M631R) & NK & 42 & {$[55,56]$} \\
\hline 89 & $\begin{array}{l}\text { Abnormal gait, speech } \\
\text { problems }(20-24)\end{array}$ & HSM & NK & Tunisian & c.1553G>A (p.R518Q) & NK & 60 & {$[55,56]$} \\
\hline
\end{tabular}

HSM: hepatosplenomegaly, VM: visceromegaly, SM: splenomegaly, PJ: prolonged jaundice, DD: developmental delay, DR: developmental regression, M: male, F: female, NK: not known, Ref: reference. 


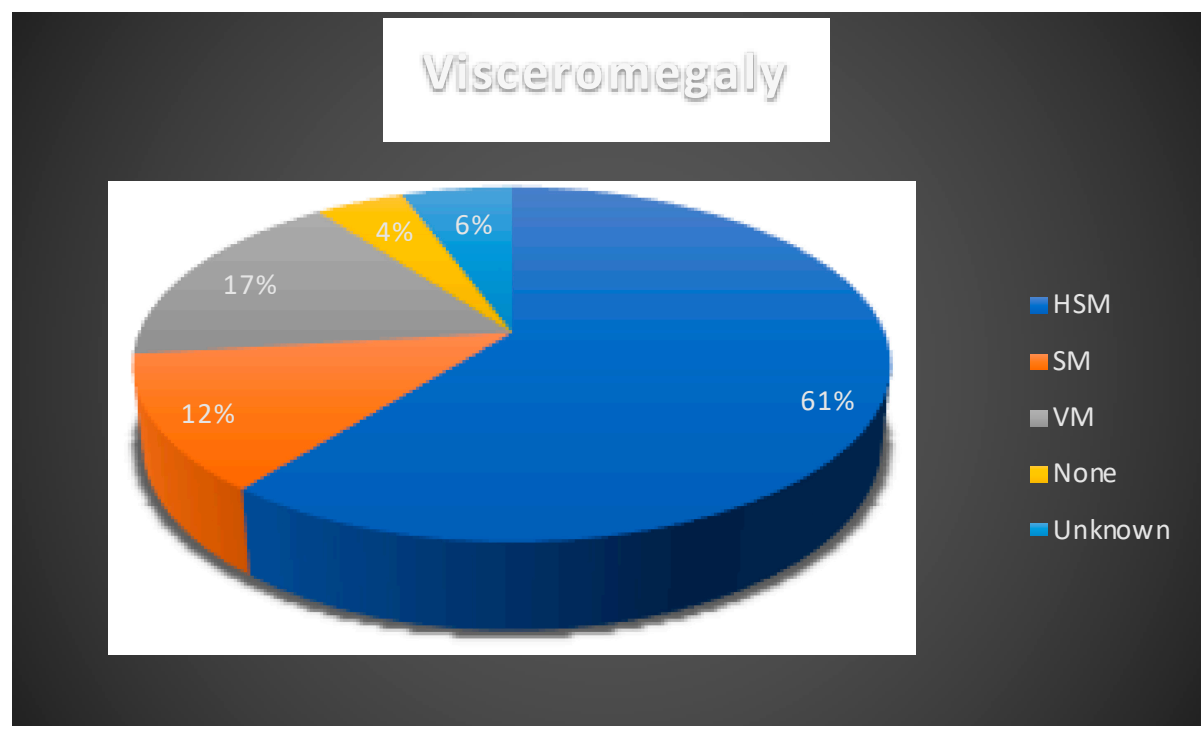

Figure 2. Presence of visceromegaly reported in NPC1 cases. HSM: hepatosplenomegaly, VM: visceromegaly, SM: splenomegaly. Blue: hepatomegaly; orange: splenomegaly; grey: visceromegaly; yellow: none; light blue: unknown

\subsection{Presentation and Progression of Neurological Symptoms}

The time of onset and the type of neurological symptoms that patients experience predict the general disease course, progression, and life span in NPC. In Table 2 and Figure 3 we detailed the initial reported neurological symptoms and/or signs and the age of their onset.

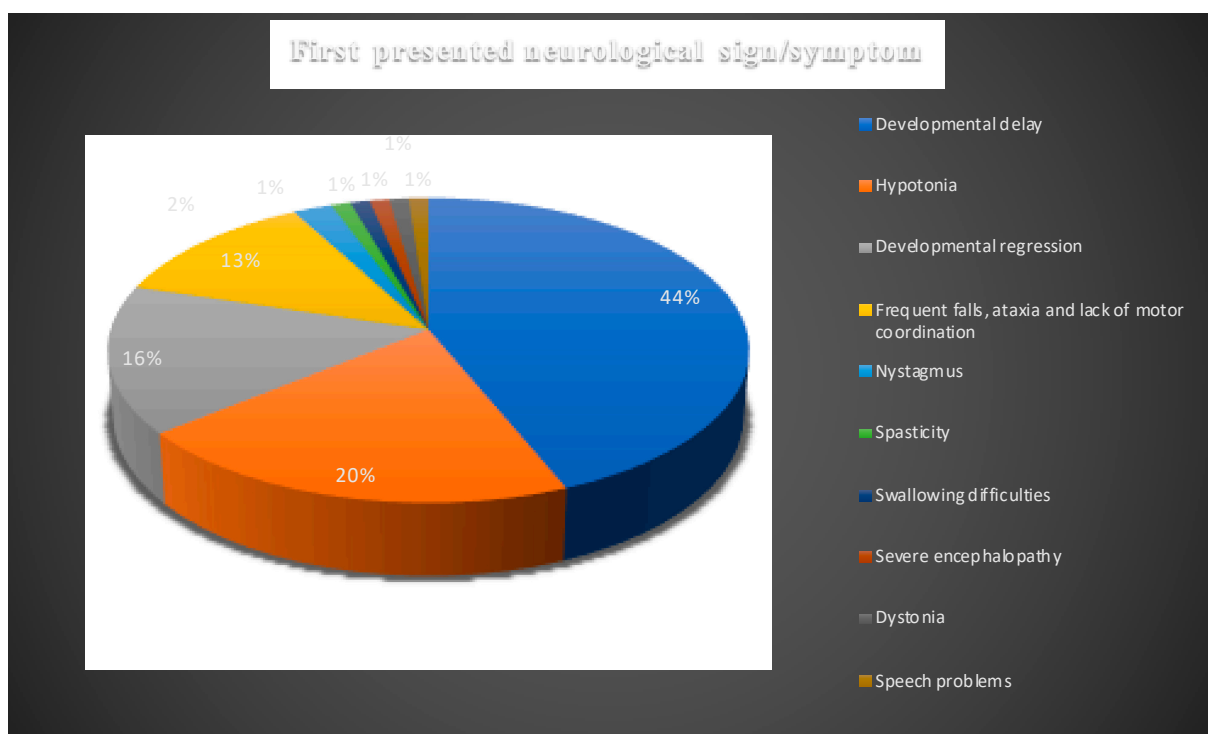

Figure 3. Presenting neurological signs and symptoms of all NPC1 patients. Blue: developmental delay; orange: hypotonia; silver: developmental regression; yellow: frequent falls, ataxia, and lack of motor coordination; light blue: nystagmus; green: spasticity; navy blue: swallowing difficulties; orange-red: severe encephalopathy; grey: dystonia; mustard: speech problems.

In order to define the typical neurological features in a better categorized cohort of patients with EIF NPC, we removed patients with very limited information, i.e., those with "developmental delay" as the only clinical characteristic, from our calculations (Table 3). 
Table 3. Range of neurological symptoms and signs. Patients selected from the total group on the basis of presence of more than one neurological symptom or sign. Patient number is linked to Table 1.

\begin{tabular}{|c|c|c|c|c|c|c|c|c|c|c|}
\hline $\begin{array}{l}\text { Patient } \\
\text { No }\end{array}$ & $\begin{array}{c}\text { Presenting Neurological } \\
\text { Sign/Symptom (Age of Onset) }\end{array}$ & Developmental Progress & $\begin{array}{c}\text { Ataxia, } \\
\text { Abnormal } \\
\text { Gait (Age } \\
\text { of Onset) }\end{array}$ & $\begin{array}{l}\text { Cataplexy } \\
\text { (Age of } \\
\text { Onset) }\end{array}$ & $\begin{array}{l}\text { Seizures } \\
\text { (Age of } \\
\text { Onset) }\end{array}$ & $\begin{array}{l}\text { VSGP } \\
\text { (Age of } \\
\text { Onset) }\end{array}$ & $\begin{array}{l}\text { Dystonia } \\
\text { (Age of } \\
\text { Onset) }\end{array}$ & $\begin{array}{c}\text { Dysphagia } \\
\text { (Age of } \\
\text { Onset) }\end{array}$ & $\begin{array}{c}\text { Dysarthria } \\
\text { (Age of } \\
\text { Onset) }\end{array}$ & $\begin{array}{l}\text { Spasticity } \\
\text { (Age of } \\
\text { Onset) }\end{array}$ \\
\hline 1 & $\begin{array}{l}\text { Swallowing difficulties, lack of } \\
\text { motor coordination }(3 \mathrm{~m})\end{array}$ & Psychomotor retardation & & & & Yes & & Yes & & Yes \\
\hline 2 & $\begin{array}{l}\text { Severe encephalopathy with } \\
\text { uncontrolled movements, ataxia, } \\
\text { tremor, nystagmus }(24 \mathrm{~m})\end{array}$ & & & & & & & & & \\
\hline 5 & Hypotonia, DD (5 m) & Motor and cognitive deficits & & & & $\begin{array}{l}\text { Yes } \\
(9 \mathrm{~m})\end{array}$ & Yes & Yes & & \\
\hline 6 & Hypotonia, DD (6 m) & Motor and cognitive deficits & & & & $\begin{array}{c}\text { Yes } \\
(18 \mathrm{~m})\end{array}$ & Yes & & & \\
\hline 7 & Hypotonia, DD (7 m) & Motor and cognitive deficits & & & & $\begin{array}{l}\text { Yes } \\
(24 \mathrm{~m})\end{array}$ & Yes & & Yes & \\
\hline 8 & Hypotonia, DD (9 m) & Motor and cognitive deficits & & & & & & & & \\
\hline 9 & Hypotonia, DD (9 m) & Motor and cognitive deficits & & & & & & & Yes & \\
\hline 10 & Hypotonia, DD (10 m) & Motor and cognitive deficits & & & & Yes & & & Yes & \\
\hline 11 & Hypotonia, DD (12 m) & Motor and cognitive deficits & & & & & & Yes & Yes & \\
\hline 12 & Hypotonia, DD (12 m) & Motor and cognitive deficits & & & & & Yes & & Yes & \\
\hline 13 & Frequent falls, DD (12 m) & Delayed motor development & & & & & & & & \\
\hline 14 & Frequent falls, DD (18 m) & $\begin{array}{c}\text { Independent walking at } 18 \mathrm{~m}, \\
\text { language delay }\end{array}$ & & $\begin{array}{l}\text { Yes } \\
(30 \mathrm{~m})\end{array}$ & & & & & & \\
\hline 15 & $\begin{array}{l}\text { Frequent falls, DD } \\
\qquad(12 \mathrm{~m})\end{array}$ & $\begin{array}{l}\text { Independent walking at } 12 \mathrm{~m} \text {, slower } \\
\text { intelligence progression, } \\
\text { psychomotor regression }\end{array}$ & & $\begin{array}{l}\text { Yes } \\
(36 \mathrm{~m})\end{array}$ & & & & & & \\
\hline 16 & $\mathrm{DD}(20 \mathrm{~m})$ & $\begin{array}{l}\text { Delayed independent walk, slow } \\
\text { motor development, psychomotor } \\
\text { regression } 24 \mathrm{~m}\end{array}$ & & & & & & & & \\
\hline 17 & Frequent falls (15 m) & Motor regression $36 \mathrm{~m}$ & & $\begin{array}{l}\text { Yes } \\
(36 \mathrm{~m})\end{array}$ & & & & & & \\
\hline 18 & Frequent falls (20 m) & & & Yes & & & & & & \\
\hline 19 & $\mathrm{DD}(\mathrm{NK})$ & Developmental delay $<24 \mathrm{~m}$ & $\begin{array}{c}\text { Yes } \\
(<24 \mathrm{~m})\end{array}$ & $\begin{array}{l}\text { Yes } \\
(24 \mathrm{~m})\end{array}$ & $\begin{array}{c}\text { Yes } \\
(48 \mathrm{~m})\end{array}$ & Yes & & $\begin{array}{l}\text { Yes } \\
(60 \mathrm{~m})\end{array}$ & Yes & \\
\hline
\end{tabular}


Table 3. Cont.

\begin{tabular}{|c|c|c|c|c|c|c|c|c|c|c|}
\hline $\begin{array}{l}\text { Patient } \\
\text { No }\end{array}$ & $\begin{array}{l}\text { Presenting Neurological } \\
\text { Sign/Symptom } \\
\text { (Age of Onset) }\end{array}$ & Developmental Progress & $\begin{array}{c}\text { Ataxia, } \\
\text { Abnormal } \\
\text { Gait (Age } \\
\text { of Onset) }\end{array}$ & $\begin{array}{l}\text { Cataplexy } \\
\text { (Age of } \\
\text { Onset) }\end{array}$ & $\begin{array}{l}\text { Seizures } \\
\text { (Age of } \\
\text { Onset) }\end{array}$ & $\begin{array}{l}\text { VSGP } \\
\text { (Age of } \\
\text { Onset) }\end{array}$ & $\begin{array}{l}\text { Dystonia } \\
\text { (Age of } \\
\text { Onset) }\end{array}$ & $\begin{array}{l}\text { Dysphagia } \\
\text { (Age of } \\
\text { Onset) }\end{array}$ & $\begin{array}{l}\text { Dysarthria } \\
\text { (Age of } \\
\text { Onset) }\end{array}$ & $\begin{array}{l}\text { Spasticity } \\
\text { (Age of } \\
\text { Onset) }\end{array}$ \\
\hline 20 & $\mathrm{DD}(\mathrm{NK})$ & $\begin{array}{l}\text { Developmental delay, never } \\
\text { mobile, no swallowing problems }\end{array}$ & & $\begin{array}{c}\text { Yes } \\
(60 \mathrm{~m})\end{array}$ & $\begin{array}{c}\text { Yes } \\
(70 \mathrm{~m})\end{array}$ & $\begin{array}{c}\text { Yes } \\
(36 \mathrm{~m})\end{array}$ & & & $\begin{array}{c}\text { Yes } \\
(<36 \mathrm{~m})\end{array}$ & \\
\hline 21 & $\mathrm{DD}(\mathrm{NK})$ & $\begin{array}{l}\text { Developmental delay, never } \\
\text { mobile, no swallowing problems }\end{array}$ & & $\begin{array}{l}\text { Yes } \\
(60 \mathrm{~m})\end{array}$ & $\begin{array}{l}\text { Yes } \\
(70 \mathrm{~m})\end{array}$ & $\begin{array}{l}\text { Yes } \\
(36 \mathrm{~m})\end{array}$ & & & $\begin{array}{c}\text { Yes } \\
(<36 \mathrm{~m})\end{array}$ & \\
\hline 22 & $\mathrm{DD}(17 \mathrm{~m})$ & Developmental delay $17 \mathrm{~m}$, ataxia & $\begin{array}{c}\text { Yes } \\
(48 \mathrm{~m})\end{array}$ & $\begin{array}{c}\text { Yes } \\
(48 \mathrm{~m})\end{array}$ & $\begin{array}{l}\text { Yes } \\
(60 \mathrm{~m})\end{array}$ & $\begin{array}{c}\text { Yes } \\
(36 \mathrm{~m})\end{array}$ & & $\begin{array}{l}\text { Yes } \\
(60 \mathrm{~m}) \\
\end{array}$ & Yes & \\
\hline 23 & $\mathrm{DD}(\mathrm{NK})$ & Developmental delay $<18 \mathrm{~m}$ & $\begin{array}{c}\text { Yes } \\
(<24 \mathrm{~m})\end{array}$ & $\begin{array}{c}\text { Yes } \\
(35 \mathrm{~m})\end{array}$ & & & & $\begin{array}{c}\text { Yes } \\
(13 \mathrm{~m})\end{array}$ & $\begin{array}{c}\text { Yes } \\
(<24 \mathrm{~m})\end{array}$ & \\
\hline 24 & $\mathrm{DD}(\mathrm{NK})$ & No speech & & & $\begin{array}{c}\text { Yes } \\
(41 \mathrm{~m})\end{array}$ & $\begin{array}{c}\text { Yes } \\
(<18 \mathrm{~m})\end{array}$ & & $\begin{array}{c}\text { Yes } \\
(36 \mathrm{~m})\end{array}$ & & \\
\hline 29 & DR, ataxia $(18 \mathrm{~m})$ & & & & & & & & & \\
\hline 30 & $\mathrm{DD}$, ataxia $(22 \mathrm{~m})$ & Psychomotor regression & & & & & & & & \\
\hline 31 & $\mathrm{DD}(12 \mathrm{~m})$ & $\begin{array}{l}\text { Developmental delay at } 1 \text { st year, } \\
\text { cannot stand and walk at } 19 \mathrm{~m}, \\
\text { tremor in upper limbs, no speech }\end{array}$ & & & $\begin{array}{l}\text { Yes } \\
(30 \mathrm{~m})\end{array}$ & & & & & $\begin{array}{c}\text { Yes } \\
(42 \mathrm{~m})\end{array}$ \\
\hline 32 & Speech regression $(18 \mathrm{~m})$ & $\begin{array}{l}\text { Early development was normal, } \\
\text { walked independently at } 14 \mathrm{~m} \text {. } \\
\text { Loss of speech at } 18 \mathrm{~m} \text {, cannot walk } \\
\text { at } 30 \mathrm{~m} \text {, left hemiparesis, could not } \\
\text { stand at } 36 \mathrm{~m} \text {, bedridden at } 48 \mathrm{~m}\end{array}$ & & & & Yes & & & Yes & \\
\hline 33 & Hypotonia (2m), DD (4 m) & $\begin{array}{c}2 \mathrm{~m} \text { hypotonia, poor sucking, } \\
\text { developmental delay (poor head } \\
\text { control at } 4 \mathrm{~m} \text { ) }\end{array}$ & & & & & & & & \\
\hline 35 & Hypotonia, DD (12 m) & Psychomotor retardation & & & & & & & & \\
\hline 37 & $\begin{array}{l}\text { Motor regression, spastic } \\
\text { tetraparesis }(12 \mathrm{~m})\end{array}$ & Motor regression & & & & & & & & \\
\hline 51 & Hypotonia $(23 \mathrm{~m})$ & Neurological regression $24 \mathrm{~m}$ & & & & & & & & \\
\hline 55 & $\mathrm{DR}(\mathrm{NK})$ & $\begin{array}{l}\text { Developmental regression, } \\
\text { intellectual disability }\end{array}$ & & & & & & & & \\
\hline 56 & DR (NK) & $\begin{array}{l}\text { Developmental regression, } \\
\text { intellectual disability }\end{array}$ & & & & & & & & \\
\hline
\end{tabular}


Table 3. Cont

\begin{tabular}{|c|c|c|c|c|c|c|c|c|c|c|}
\hline $\begin{array}{l}\text { Patient } \\
\text { No }\end{array}$ & $\begin{array}{l}\text { Presenting Neurological } \\
\text { Sign/Symptom } \\
\text { (Age of Onset) }\end{array}$ & Developmental Progress & $\begin{array}{c}\text { Ataxia, } \\
\text { Abnormal } \\
\text { Gait (Age } \\
\text { of Onset) }\end{array}$ & $\begin{array}{l}\text { Cataplexy } \\
\text { (Age of } \\
\text { Onset) }\end{array}$ & $\begin{array}{l}\text { Seizures } \\
\text { (Age of } \\
\text { Onset) }\end{array}$ & $\begin{array}{l}\text { VSGP } \\
\text { (Age of } \\
\text { Onset) }\end{array}$ & $\begin{array}{l}\text { Dystonia } \\
\text { (Age of } \\
\text { Onset) }\end{array}$ & $\begin{array}{l}\text { Dysphagia } \\
\text { (Age of } \\
\text { Onset) }\end{array}$ & $\begin{array}{l}\text { Dysarthria } \\
\text { (Age of } \\
\text { Onset) }\end{array}$ & $\begin{array}{l}\text { Spasticity } \\
\text { (Age of } \\
\text { Onset) }\end{array}$ \\
\hline 57 & $\mathrm{DR}(\mathrm{NK})$ & $\begin{array}{l}\text { Developmental regression, } \\
\text { intellectual disability, hearing } \\
\text { impairment, visual impairment }\end{array}$ & & & & Yes & & & & \\
\hline 58 & DR (NK) & Developmental regression & & & Yes & & & & & \\
\hline 59 & $\mathrm{DD}$, ataxia (24) & Mild global developmental delay & & & & $\begin{array}{l}\text { Yes } \\
(54 \mathrm{~m})\end{array}$ & & & $\begin{array}{c}\text { Yes } \\
(54 \mathrm{~m})\end{array}$ & \\
\hline 75 & $\mathrm{DD}(5)$ & $\begin{array}{l}\text { Head control 5m, walking } \\
\text { independently } 24 \mathrm{~m}\end{array}$ & $\begin{array}{c}\text { Yes } \\
(30 \mathrm{~m})\end{array}$ & & & $\begin{array}{c}\text { Yes } \\
(45 \mathrm{~m})\end{array}$ & $\begin{array}{c}\text { Yes } \\
(45 \mathrm{~m})\end{array}$ & $\begin{array}{c}\text { Yes } \\
(36 \mathrm{~m})\end{array}$ & $\begin{array}{c}\text { Yes } \\
(36 \mathrm{~m})\end{array}$ & $\begin{array}{c}\text { Yes } \\
(45 \mathrm{~m})\end{array}$ \\
\hline 76 & $\mathrm{DD}, \mathrm{DR}(9)$ & $\begin{array}{l}\text { Developmental regression } 9 \mathrm{~m}, \\
\text { never walk, no speech }\end{array}$ & Yes & Yes & Yes & Yes & & Yes & & Yes \\
\hline 77 & Hypotonia, DD (NK) & $\begin{array}{l}\text { Mild psychomotor retardation, } \\
\text { decreased muscular tone, } \\
\text { walking difficulties }\end{array}$ & & & & & & & & \\
\hline 78 & $\begin{array}{l}\text { Hypotonia, DD, } \\
\text { Dystonia (5) }\end{array}$ & $\begin{array}{c}\text { Able to sit: } 18 \mathrm{~m} \\
\text { Never stand up or walk }\end{array}$ & & & & $\begin{array}{l}\text { Yes } \\
(8 \mathrm{~m})\end{array}$ & & & & \\
\hline 82 & DD, DR (12) & & & & & & & & & \\
\hline 83 & DD, DR (24) & & & & & & & & & \\
\hline 84 & $\mathrm{DD}, \mathrm{DR}(24)$ & & & & & & & & & \\
\hline 85 & DD, DR (18) & & & & & & & & & \\
\hline
\end{tabular}


This narrowed the cohort down to 43 patients with at least two neurological signs or symptoms. Developmental delay remained the most common first reported symptom which was noted in $31 / 43(72 \%)$. Developmental delay was noticed in $17 / 43$ of cases in the first year of life and 8/43 between 13 and 24 months of age. In six patients age of onset of the delay was not reported.

Hypotonia was the second most frequently reported initial symptom which was recorded in $13 / 43$ patients. While it was reported in $11 / 43$ patients in first 12 months of life, $1 / 43$ patients presented with hypotonia between 13 and 24 months (Figure 3). In one case, the age onset of hypotonia was not identified. In 12 cases, hypotonia was noted to accompany developmental delay.

A total of $12 / 43$ cases were reported to have exhibited developmental regression which means losing acquired functions or failing to progress after a normal developmental period, indicating a neurodegenerative disorder. Regression was described to occur in the first year of life in 3/43 patients, in the second year of life in 5/43 patients, and was not defined in $4 / 43$.

Frequent falls, lack of motor coordination, and ataxia were reported as a presenting symptom in 10/43 patients; $3 / 10$ in the first 12 months and $7 / 10$ between 13 and 24 months. Frequent falls and ataxia were associated with developmental delay in $5 / 10$ patients.

Spasticity, dystonia, and dysphagia were the other initial neurological symptoms described; each was reported in one patient occurring in the first 12 months of age.

Nystagmus was noted as a presenting symptom in one patient accompanied by severe encephalopathy with uncontrolled movements, ataxia, and tremor at the age of 24 months.

Descriptions of these 43 patients had varying degrees of detail regarding the neurological progression of the disease. Age distribution of neurological symptoms and signs is shown in Figure 4. In 7/43 patients, additional symptoms were described in the first 24 months of life: VSGP in five cases, ataxia, dysphagia, and dysarthria in one case and one patient was described to display both ataxia and cataplexy. Most additional symptoms were described to have occurred after 24 months of age.

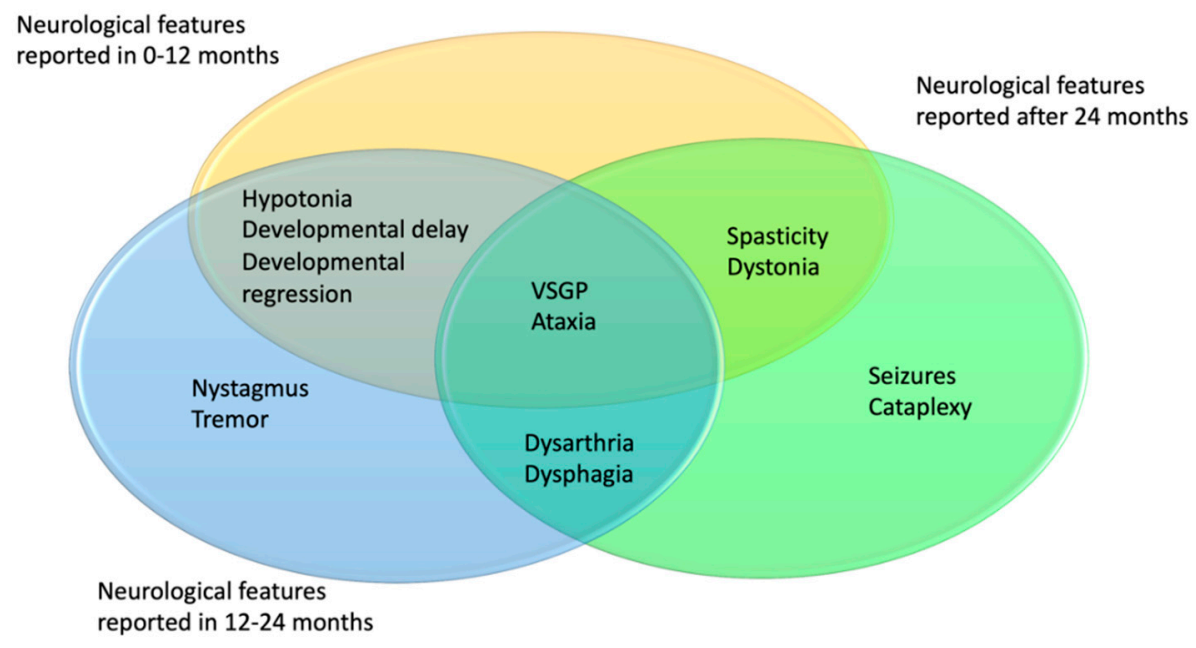

Figure 4. Distribution of age of onset for the neurological symptoms in NPC1 patients. In yellow 0-12 months, blue 13-24 months, green 24+ months.

Vertical supranuclear gaze palsy and dysarthria were the most common neurological manifestations developed in the course of the disease; $16 / 43(37.2 \%)$ and $13 / 43(30.2 \%)$, respectively. VSGP was typically reported between 8 and 54 months.

A total of 10/43 cases developed cataplexy, which is a sudden and transient episode of muscle weakness and loss of consciousness lasting a few seconds and followed by full conscious awareness. The onset of cataplexy was described to have occurred between the ages of $2-5$ years. A total of five of them were determined as gelastic cataplexy which is triggered by laughter.

Dysphagia and swallowing difficulties were reported in $9 / 43$ patients between the ages of 13 and 60 months. A total of $8 / 43$ patients developed seizures between the ages of 30 and 70 months. 
Ataxia/abnormal gait was described in 5/43 patients during the follow-up. A total of $4 / 5$ patients with ataxia were also reported to have dysarthria. Dystonia was described in $5 / 43$ cases and spasticity in $4 / 43$.

\subsection{Developmental Milestones}

Delay in reaching normal developmental milestones is the major presenting feature of EIF NPC. Unfortunately, little detail was provided in the description of the milestone achievement and therefore it was not possible to tease out specific problems in most cases.

The distribution of description of developmental delay is shown in Figure 5. Developmental delay was reported in 31/43 patients. Motor development delay was the most commonly mentioned abnormality among the EIF patients, reported in 20/43 patients. In 10/43 patients motor delay was accompanied by cognitive delay and in $3 / 43$ patients by speech delay. In $1 / 43$ case only speech delay was mentioned. Unspecified developmental delay was mentioned in 9/43 patients and global development delay was noted in $1 / 43$ patients. Hearing and visual impairment were reported in one patient.

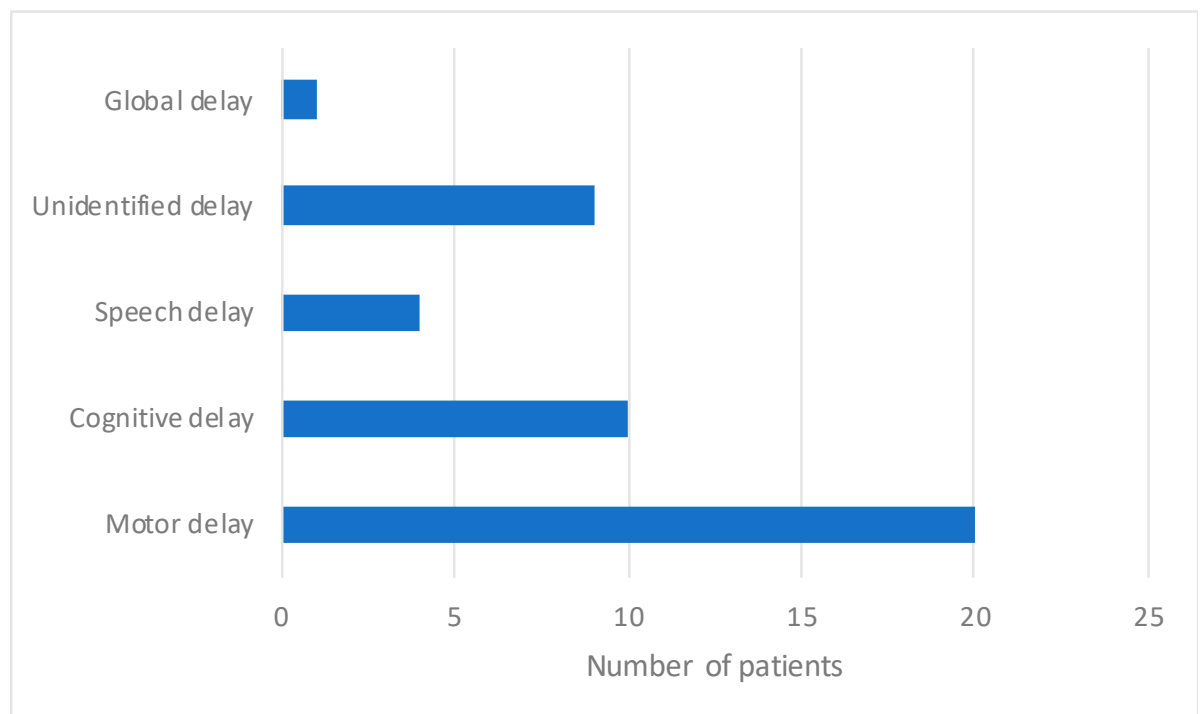

Figure 5. Distribution of reported developmental delay in NPC1 patients.

\subsection{Age and Cause of Death}

Age of death was reported in 31/89 patients. Median age of death was 48 months (range 7-132 months). Cause of death was only reported in 7/31 cases and all of them were due to respiratory failure. A total of 3/31 of these patients received miglustat treatment. A total of two of them started miglustat therapy at the age of 7 years, however they only received treatment for 1 week. One other patient started miglustat therapy at the age of 20 months and after 13 months of therapy, the patient was deceased due to the respiratory complications.

\subsection{Neuroimaging Findings}

Neuroimaging data was available in 10/81 patients and only one of them was reported as normal [51]. Cerebral atrophy was mentioned in nine patients [42,46,49,50]. Deep white matter signal abnormalities were seen in six patients [42,49].

\subsection{Additional Diagnostic Investigations}

The filipin test, based on the demonstration of accumulated unesterified cholesterol within the lysosomes of cultured fibroblasts, for many years was regarded as the gold standard for NPC diagnosis, however, more recently it has been used less frequently compared with analysis of other biomarkers [12]. 
As expected, most of the historical patients underwent filipin staining. Filipin test was used to confirm the diagnosis in patients in whom only a single NPC1 mutation was identified. The other tests that were commonly employed to assist in the diagnostic process in the past included bone marrow aspiration and chitotriosidase activity assay. These analyses are not recommended anymore as they are not as sensitive or specific as the newer tests such as analyses of oxysterols, NPC bile acids, and lyso-SM-509 levels [3].

\subsection{Genotype Phenotype Correlation}

In order to identify NPC1 mutations that confer EIF phenotype we selected 42 patients with at least two neurological signs or symptoms from Table 2. As above, patients who presented with only mild developmental delay were not included in order to avoid potential inclusion error (Table 2). We were able to identify mutations that in homozygous state confer EIF phenotype (Table 3). Some of these mutations were also seen in homozygous state in LIF phenotype suggesting a degree of overlap between the two groups. A combination of the severe (EIF causing) mutation with a milder one was seen to confer the later onset disease.

A total of 15 mutations occurred in homozygous state in EIF patients (Table 4). They included two gross deletions, one small deletion, two insertions and deletions that result in a frame shift, two splice site mutations, and eight missense mutations.

A total of 9/15 mutations: deletion of exon 1+promoter, c.3578_3591 + 9del, c.385delT, p.C63fsX75, IVS21-2delATGC, с. 2740T>A (p.C914S), c.3584G>T (p.G1195V), c.3478-6T>A, c.960_961dup (p.A321Gfs*16) were only reported in EIF patients.

c.3503G > A (p.C1168Y) was identified in one EIF patient but also in two LIF patients in a homozygous state $[37,55]$

c.1415T>C (p.L472P) in exon 9 of the NPC1 gene was found in two Iranian patients with EIF [53]. It was previously reported in two other Iranian patients in a homozygous state; one of them was also EIF patient, while the other was a LIF patient [45].

c.3394G >C (p.A1132P), a missense point mutation in exon 22, was reported in a Greek patient with an EIF. The same report described a LIF patient who died at the age of 14 years with the same mutation [13].

Homozygous gross deletion of exon 1+promoter was reported in one patient [48]. In previous reports, three patients were also described with a EIF phenotype even though they had different gross deletions of the NPC1 gene in a compound heterozygous state [39,57].

c.1211G >A (p.R404Q) was found in Bedouin-Israeli patients in homozygous state who had EIF, LIF, and EISL phenotypes [47]. It was also detected in a French EIF patient in a compound heterozygous state with c.709C $>$ T (p.237S) [56]. A total of two juvenile patients were reported to have c.1211G $>A$ (p.R404Q); one as the only mutation, the other was in combination with c.1133T >C (p.Vl378A) [37].

c.3104C $>$ T (p.A1035V) was reported in two EIF patients; one in homozygous state, the other was reported in combination with IVS23+1G $>$ A [52]. It was also reported in a juvenile patient with an unknown second allele [52]. Another juvenile patient had c.3104C $>$ T (p.A1035V) in combination with c.3019C $>$ G (p.P1007A), again suggesting that this latter mutation confers milder phenotype [58].

Besides occurring in homozygous state, there were two patients referred in literature being compound heterozygotes for IVS23 +1G > A (c.3591+1G >A) with c.3104C $>$ T (p.A1035V) or c.3182T $>C$ (p.I1061T), respectively, who displayed the EIF phenotype and another patient who had this mutation in combination with c.2090C > T (p.V697A) and showed the LIF phenotype, confirming the hypothesis that this mutation confers more severe illness [36,52,59].

c.3107C $>\mathrm{T}$ (p.T1036M) is a severe mutation which was associated with EIF phenotype in a homozygous state [42]. It was reported in two other EIF patients in combination with c.3557G $>\mathrm{A}$ (p.R1168H) [37]. c.3107C > T (p.T1036M) was found in two LIF and one juvenile patient, in combination with c.3182C > T (p.I1061T) and c.2861C > T (p.S954L) mutations that typically confer later onset phenotypes [37]. 
Table 4. NPC1 mutations causing early infantile phenotype. Mutation-phenotype association.

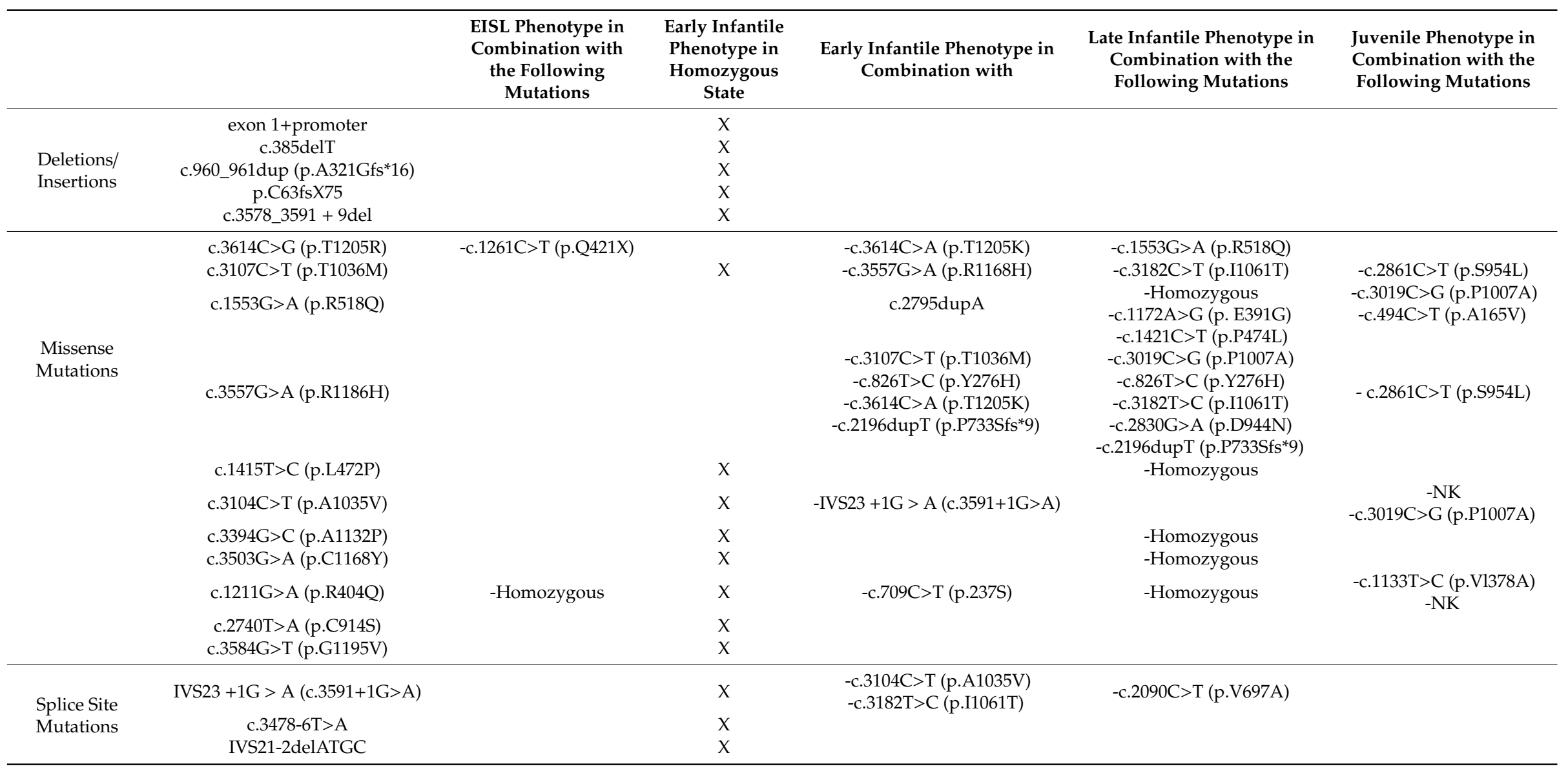


c.3557G >A (p.R1186H) mutation was found in the EIF phenotype when it occurs in combination with c.3107C > T (p.T1036M), c.826T>C (p.Y276H), c.3614C >A (p.T1205K), and c.2196dupT (p.P733Sfs*9) [36,37]. It was also reported in LIF patients in combination with c.1421C>T (p.P474L), c.3019C >G (p.P1007A), c.826T>C (p.Y276H), c.3182T>C (p.I1061T), c.2830G >A (p.D944N), and c.2196dupT (p.P733Sfs*9) [36,60]. c.3557G $>$ A (p.R1186H) mutation was also detected in juvenile patients when it occurs in combination with a c.2861C $>$ T (p.S954L) mutation known to confirm juvenile phenotype $[36,60]$. c.3557G $>$ A (p.R1186H) was found in a LIF patient and a patient with isolated splenomegaly without neurological presentation in a homozygous state, which suggests that the severity of this mutation depends on the additional genomic factors [36].

While c.1553G >A (p.R518Q) was found in two EIF patients in combination with c.2795dupA, it was detected in a LIF patients in a homozygous state and in combination with c.1172A $>\mathrm{G}$ (p. E391G) [61,62]. It was shown in juvenile patients in combination with c.3019C $>$ G (p.P1007A) and c.494C > T (p.A165V) $[37,61]$.

While c.3614C $>$ G (p.T1205R) was reported in an EIF patient with c.3614C >A (p.T1205K), it was also noted in a LIF patient in a combination with c.1553G $>$ A (p.R518Q) suggesting that the latter mutation confers milder phenotype [63]. It was detected in a patient presented as an EISL phenotype in a compound heterozygous state with c.1261C $>$ T (p.Q421X) [36].

\subsection{Early Infantile Neurological Onset NPC Due to NPC2 Mutations}

Analysis of massively parallel sequencing data sets revealed that incidence rate for NPC2 mutations is extremely rare at 1/2,858,998 [16]. Therefore, only a few small case series were reported [64-68].

An observational, retrospective study of NPC patients from Czech Republic revealed 56 cases and only one of them had NPC2 genotype with an EIF phenotype. This female patient presented with pulmonary involvement, psychomotor retardation, central hypotonia, and moderate hepatosplenomegaly at the age of 1 year. Spasticity and cognitive deterioration occurred during the second year of life and the patient died at 4 years of age due to respiratory insufficiency [36].

Retrospective data for UK-based patients with NPC revealed two cases with NPC2 mutations among a total of 112 patients. Both of them were LIF patients with few insidious neurological manifestations [37].

A collaborative multicenter study from Italy which aimed to characterize the molecular basis of NPC reported eight NPC2 patients, six of whom presented with severe phenotypes including EISL and EIF; two patients had an adult phenotype [41].

The clinical, biochemical, and molecular findings of 14 NPC cases diagnosed in Greece were demonstrated and only 1/14 had NPC2 mutations causing a LIF [54].

A prospective epidemiologic cohort study from Turkey aimed to investigate the frequency of NPC mutations in consanguineous families with at least one homozygous family member. A total of 3/4 randomly selected probands had NPC2 mutations, which is likely to demonstrate a founder effect in this region [67].

According to the review of literature, 16 NPC2 cases were found with EIF (Table 5). Cases which did not have neurological symptoms were excluded. A total of $8 / 16$ cases were female, 3/16 were male, and $5 / 16$ were unknown gender.

While visceral symptoms were found in all patients, visceromegaly was noted in 15/16 patients (93.8\%); 9/16 had HSM, 4/16 were SM, and 2/16 had HM. Pulmonary involvement was reported in 12/16 patients. Prolonged jaundice was noted in 7/16 patients.

Age of neurological onset was reported in 14 patients with median age of onset 7.5 months (range 0-24). Hypotonia and developmental delay were the most common initial neurological symptoms, reported in 10/16 and 8/16 cases, respectively. While hypotonia presented in 8/16 patients in the first 12 months, it was reported to have occurred between 12 and 24 months in 2/16 patients. Similar to that developmental delay was noted in 6/16 patients in the first year of life and 2/16 cases in the second year of life. In one case, abnormal gait accompanied hypotonia as a presenting neurological symptom. 
Table 5. Demographic, clinical, and molecular features of early infantile disease with NPC2 mutations.

\begin{tabular}{|c|c|c|c|c|c|c|c|c|}
\hline Patient No & $\begin{array}{c}\text { Presenting Neurological } \\
\text { Sign/Symptom (Age of Onset } \\
\text { in Months) }\end{array}$ & $\begin{array}{c}\text { Visceral } \\
\text { Symptoms }\end{array}$ & Ethnicity & $\begin{array}{l}\text { Sex } \\
\text { F/M }\end{array}$ & Allele 1 & Allele 2 & $\begin{array}{c}\text { Age of } \\
\text { Death } \\
\text { (Month) }\end{array}$ & Ref. \\
\hline 1 & $\mathrm{DD}(12)$ & HSM, PI & Czech & $\mathrm{F}$ & c.58G > T (p.E20X) & c.58G > T (p.E20X) & 48 (RF) & [36] \\
\hline 2 & NK & HSM, PI & Italian & NK & c.58G > T (p.E20X) & c.58G > T (p.E20X) & $10(\mathrm{RF})$ & [40] \\
\hline 3 & NK & HSM, PI & Italian & NK & c.58G > T (p.E20X) & c.58G > T (p.E20X) & NK & [40] \\
\hline 4 & DD, Hypotonia (12) & $\mathrm{SM}$ & Turkish & $\mathrm{F}$ & c.352G > T (p.E118X) & c.352G > T (p.E118X) & $24(\mathrm{RF})$ & [67] \\
\hline 5 & Hypotonia & PJ, HSM, PI & Turkish & $\mathrm{F}$ & c.352G > T (p.E118X) & c.352G > T (p.E118X) & $10(\mathrm{RF})$ & [67] \\
\hline 6 & Hypotonia, DD (2) & HSM, PJ, PI & Turkish & $\mathrm{F}$ & c.434T>A (p.V145E) & c. $434 \mathrm{~T}>\mathrm{A}(\mathrm{p} . \mathrm{V} 145 \mathrm{E})$ & $8(\mathrm{RF})$ & [68] \\
\hline 7 & Newborn Hypotonia (NK) & HSM, PJ, PI & Turkish & $\mathrm{F}$ & c. $434 \mathrm{~T}>\mathrm{A}(\mathrm{p} . \mathrm{V} 145 \mathrm{E})$ & c. $434 \mathrm{~T}>\mathrm{A}(\mathrm{p} . \mathrm{V} 145 \mathrm{E})$ & 9 (RF) & {$[67]$} \\
\hline 8 & Hypotonia (4) & SM & Turkish & NK & c.352G > T (p.E118X) & c.352G > T (p.E118X) & NK & {$[67]$} \\
\hline 9 & Hypotonia (2) & SM, PJ, PI & Tunisian & $\mathrm{M}$ & c.436C > T (p.Q146X) & c.436C > T (p.Q146X) & $4.5(\mathrm{RF})$ & [65] \\
\hline 10 & Hypotonia, DD (8) & PI, HSM & German & $\mathrm{F}$ & c.352G > T (p.E118X) & c.352G > T (p.E118X) & $11(\mathrm{RF})$ & [65] \\
\hline 11 & $\mathrm{DD}(7)$ & PJ, PI & Algerian & M & c.58G > T (p.E20X) & c.58G > T (p.E20X) & 19 (RF) & {$[64,69]$} \\
\hline 12 & Hypotonia, abnormal gait (18) & HSM, PI & Turkish & $\mathrm{F}$ & c.199T>C (p.S67P) & c.199T>C (p.S67P) & Alive (45y) & {$[64]$} \\
\hline 13 & $\mathrm{DD}(16)$ & HSM, PJ & Italian & NK & c.133C > T (p.Q45X) & c.133C > T (p.Q45X) & Alive (54) & [70] \\
\hline 14 & Hypotonia, DD (1) & HM, PJ, PI & Sri-Lankan & NK & c.141C >A (p.C47X) & c. $141 \mathrm{C}>\mathrm{A}(\mathrm{p} . \mathrm{C} 47 \mathrm{X})$ & Alive (12) & [70] \\
\hline 15 & Hypotonia (12) & HM, PI & Indian & $\mathrm{M}$ & c. $82+2 \mathrm{~T}>\mathrm{C}(\mathrm{IVS} 1+2 \mathrm{~T}>\mathrm{C})$ & c. $82+2 \mathrm{~T}>\mathrm{C}(\mathrm{IVS} 1+2 \mathrm{~T}>\mathrm{C})$ & NK & [71] \\
\hline 16 & $\mathrm{DD}(12)$ & SM & Chinese & $\mathrm{F}$ & c.3G $>C$ (p.M1I) & c. $190+5 \mathrm{G}>\mathrm{A}(\mathrm{IVS} 2+5 \mathrm{G}>\mathrm{A})$ & NK & [46] \\
\hline
\end{tabular}

HSM: hepatosplenomegaly, VM: visceromegaly, SM: splenomegaly, PJ: prolonged jaundice, DD: developmental delay, PI: pulmonary involvement, M: male, F: female, NK: not known, 
There was not enough data to detail the neurological progression and developmental milestones in NPC2 patients, mainly because most succumb in infancy. Distribution of neurological symptoms are shown in Figure 6. A total of three patients exhibited hypotonia with developmental delay during the follow-up. Dysarthria was reported in two cases. While VSGP was mentioned in one case, another patient developed mild horizontal saccade abnormalities and end-point nystagmus as well as intermittent myoclonus. Ataxia and cataplexy were reported in the same patient. One patient was reported to develop intellectual regression and another developed seizures at the age of 7 years.

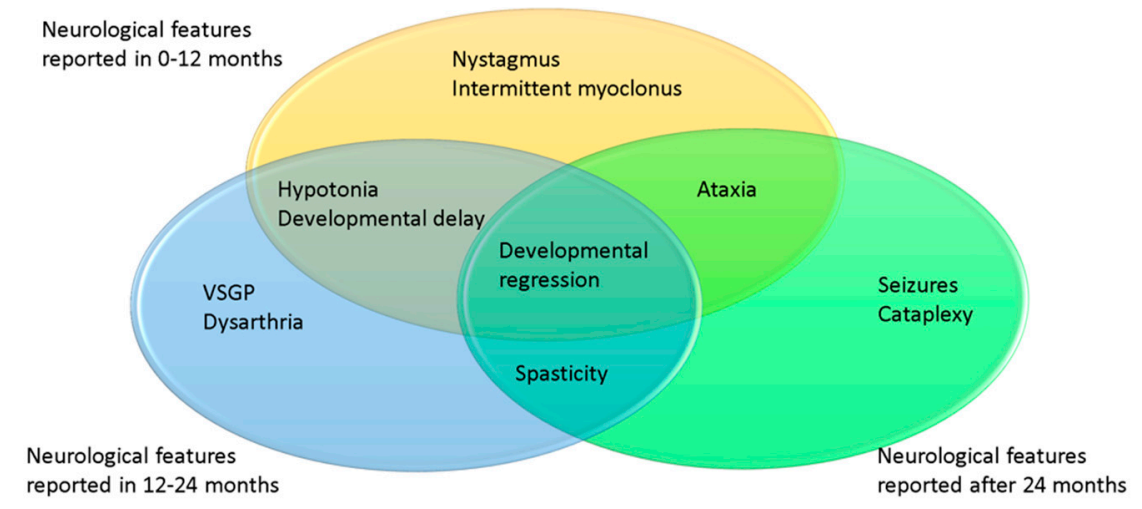

Figure 6. Age of onset of presentation for neurological symptoms in NPC2 patients. In yellow 0-12 months, blue 12-24 months, green 24+ months.

A total of nine patients were reported as deceased with the median age of 10 months (range 4.5-48). In all nine patients, cause of death was reported as respiratory failure.

NPC2 mutations causing EIF are shown in Table 6, based on mutation-phenotype association. c.58G $>\mathrm{T}$ (p.E20X) nonsense mutation was the most common reported in five cases in a homozygous state. c.58G $>$ T creates a premature stop codon downstream of the signal peptide. All patients had early onset pulmonary involvement with neurological deterioration $[36,40,64]$. However, two other patients homozygous for the same mutation presented with hepatosplenomegaly without reported neurological symptoms, significant respiratory disease, and death at the age of 6 months [64]. It was also associated with respiratory failure and premature death without obvious neurological involvement in a French patient in combination with c.27delG (p.Leu10Serfs*25) [64]. It was also demonstrated in a LIF patient in a homozygous state [37].

c.352G $>\mathrm{T}$ (p.E118X) was the second most frequent mutation observed in four patients in a homozygous state. This nonsense mutation in exon 3 of NPC2 leads to a premature stop codon and was associated with a severe clinical progression and death in the first 2 years of life $[65,67]$. It was reported in a German patient presented with visceromegaly, respiratory involvement, and early death without assigned neurological symptoms [64].

c.434T $>$ A (p.V145E) was exclusively reported in two patients with EIF in a homozygous state.

The following mutations: c.436C > T (p.Q146X), c.199T>C (p.S67P), c.133C > T (p.Q45X), c.141C >A (p.C47X), and c.82+2T>C (IVS1+2T $>$ C) were all reported in individual cases. All patients had severe pulmonary disease accompanied by neurological involvement. Homozygous c.436C >T (p.Q146X) was also reported in an Algerian patient with cholestatic icterus and hepatosplenomegaly with ascites $[66,72]$. c. $82+2 \mathrm{~T}>\mathrm{C}$ (IVS1+2T>C) was also demonstrated in a Sri-Lankan patient in whom hepatosplenomegaly, severe pulmonary involvement with hypoxia, and severe nutritional problems were first detected aged 4.5 months without obvious neurological involvement [66].

c.434T>A (p.V145E), c.199T>C (p.S67P), c.133C > T (p.Q45X), c.141C >A (p.C47X) mutations were only associated with EIF.

One Chinese patient was found to be compound heterozygous for c.3G $>C$ (p.M1I) and c.190+5G $>A$ (IVS2+5G $>$ A) mutations [46]. While c.3G $>$ C (p.M1I) was only reported in this patient, c.190+5G $>$ A (IVS2+5G $>A$ ) was reported in two juvenile cases in a homozygous state [54,64]. 
Table 6. NPC2 mutations causing early infantile phenotype. Mutation-phenotype association.

\begin{tabular}{|c|c|c|c|c|c|c|}
\hline & & $\begin{array}{l}\text { EISL Phenotype in } \\
\text { Combination with the } \\
\text { Following Mutations }\end{array}$ & $\begin{array}{l}\text { Early Infantile } \\
\text { Phenotype in } \\
\text { Homozygous } \\
\text { State }\end{array}$ & $\begin{array}{l}\text { Early Infantile } \\
\text { Phenotype in } \\
\text { Combination with } \\
\text { the Following } \\
\text { Mutations }\end{array}$ & $\begin{array}{l}\text { Late Infantile } \\
\text { Phenotype in } \\
\text { Combination with } \\
\text { the Following } \\
\text { Mutations }\end{array}$ & $\begin{array}{c}\text { Juvenile Phenotype } \\
\text { in Combination with } \\
\text { the Following } \\
\text { Mutations }\end{array}$ \\
\hline \multirow{8}{*}{ Missense Mutations } & c.58G > T (p.E20X) & $\begin{array}{c}\text {-Homozygous } \\
\text {-c.27delG (p.L10Sfs*25) }\end{array}$ & $x$ & & -Homozygous & \\
\hline & c.352G > T (p.E118X) & -Homozygous & $x$ & & & \\
\hline & c.434T>A (p.V145E) & & $x$ & & & \\
\hline & c. $133 \mathrm{C}>\mathrm{T}$ (p.Q45X) & & $X$ & & & \\
\hline & c.141C>A (p.C47X) & & $X$ & & & \\
\hline & c.199T>C (p.S67P) & & $x$ & & & \\
\hline & c.436C>T (p.Q146X) & -Homozygous & $x$ & & & \\
\hline & c.3G>C (p.M1I) & & & c. $190+5 \mathrm{G}>\mathrm{A}(\mathrm{IVS} 2+5 \mathrm{G}>\mathrm{A})$ & & \\
\hline Splice Site & $\begin{array}{c}\text { c. } 82+2 \mathrm{~T}>\mathrm{C}(\mathrm{IVS} 1+2 \mathrm{~T}>\mathrm{C}) \\
\text { c. } 190+5 \mathrm{G}>\mathrm{A}(\mathrm{IVS} 2+5 \mathrm{G}>\mathrm{A})\end{array}$ & -Homozygous & $x$ & $-\mathrm{c} .3 \mathrm{G}>\mathrm{C}(\mathrm{p} \cdot \mathrm{M} 1 \mathrm{I})$ & & -Homozygous \\
\hline
\end{tabular}




\section{Discussion}

This review intended to consolidate the knowledge of the EIF NPC clinical presentation in order to provide an up-to-date evaluation of the characteristics of this form of the disease. Granular understanding of the clinical symptom progression is of paramount importance to good clinical practice, accurate family counselling, and clinical trial design. A major limitation of this review based on literature search was the considerable heterogeneity among the included studies, particularly the way patient demographic and clinical characteristics were reported. Moreover, neurological data including the chronology of signs and symptoms and history of developmental progression were very limited. Therefore, in order to make the most reliable and accurate conclusions, we restricted the patient cohort and selected the cases with more detailed information in a consistent way. Several exclusion criteria were set to minimize the possibility of bias. Hence, clinical characteristics including the initial presentation, neurological and developmental progression, and molecular features of both EIF NPC1 and NPC2 group were set.

As expected, a remarkable proportion of EIF patients had a history of visceromegaly (80/89). However, only 30/89 patients had prolonged neonatal jaundice reported [1,36-38,46]. The presence of these symptoms and early referral to the treatment center is likely to allow early diagnosis of NPC, provided appropriate diagnostic measures are instigated [12,30,73].

Lung involvement is a prominent feature of the NPC2 disease. It is thought to be caused by the lung infiltration by foamy macrophages and is associated with pulmonary alveolar proteinosis, unlike NPC1 patients in whom respiratory complications are typically due to recurrent aspirations and infections $[47,74-80]$.

Failure to progress along the expected developmental milestones is usually the first opportunity to identify the neurological abnormality in a patient with EIF NPC [2]. Onset of neurological symptoms predicts the disease progression and the expected lifespan [1,12]. In the EIF group with two or more neurological symptoms the median age of onset was 12 months (range 0-24) in the NPC1 and 7.5 months (range 0-24) in the NPC2. The most frequent neurological symptoms were developmental delay and hypotonia in both groups. NPC1 patients also displayed developmental regression, "frequent falls and ataxia", spasticity, dysphagia, and nystagmus as an initial neurological symptom. Both hypotonia and developmental delay occurred in the first year of life more often than in the second 12 months.

From the reviewed data it was difficult to obtain granularity in the developmental delay data (Figure 5). Whilst this symptom is crucial for early diagnosis of EIF, it is well known that cases with LIF and even juvenile types of the disease may display some developmental delay. Hence, in this review we tried to select patients with features that provided more reassurance regarding the specific NPC form. Nevertheless, it is difficult to be absolutely confident in this selection. Furthermore, as an isolated symptom developmental delay leads to a large list of differential diagnoses and hence may not be helpful in the diagnostic process.

The age of presentation of various neurological symptoms is shown in Figures 4 and 6. VSGP and dysarthria were the most common followed by cataplexy, dysphagia, seizures, ataxia, and spasticity. Gelastic cataplexy and VSGP have been reported as highly specific for NPC and in particular VSGP is often suggested as the initial neurological sign. Hence, we were keen to explore whether this statement holds true for the EIF $[30,73,81]$. In the cohort with at least two neurological signs or symptoms, we found that VSGP was reported in 16/43 and mostly developed earlier (median 30 months, range 8-54 months) than cataplexy, which was reported in 10/43 cases (median 36, range 24-60 months). Thus, ophthalmological assessment is an important part of the follow-up management but absence of VSGP as a presenting feature cannot exclude EIF type of NPC.

Brain imaging in NPC is usually nonspecific early in the disease and is often reported as cerebellar and cerebral atrophy at later stages of progression. In the EIF cohort reviewed here we found that brain atrophy and white matter signal abnormalities were the most frequent changes reported [42,49-51] consistent with other studies [82]. 
We have not specifically interrogated the data on laboratory investigations performed in this cohort. Typically filipin staining and other biomarker analyses such as levels of oxysterols, lysoSM509 ratio, NPC specific bile acids are used for diagnostic screening, whilst molecular tests provide the definitive result in most, although not in all, cases [3,15,17].

According to the literature, the EIF patients live shorter after the onset of neurological disease than those patients with later presentations $[1,18,20,37,83]$. Median age of death was earlier in the NPC2 (median 10 months, range 4.5-48) compared to NPC1 patients (median 48 months, range 7-132). In agreement with individual reports, respiratory complications were the most frequent cause of death in EIF patients in both NPC1 and NPC2 groups. The earlier death may be due to the more severe pulmonary involvement in NPC2 cases. $[64,66,67,70]$. NPC1 patients usually die following the neurological disease progression, leading to respiratory complications such as aspiration pneumonia and respiratory failure [84].

We interrogated professional version of the Human Gene Mutation Database (www.hgmd.cf.ac.uk) as an up-to-date source of disease mutations in order to verify our findings from the literature review. It lists a total of 524 NPC1 defects that include 344 missense and nonsense mutations, 73 small deletions, 47 small insertions, 43 mutations affecting splicing, 12 gross deletions, three small indels, and two gross insertions/duplications. The NPC1 gene encodes NPC1 protein has 13 transmembrane domains, a sterol-sensing domain, a cysteine rich luminal loop, and a highly conserved domain with a leucine-zipper motif in the N-terminal tail. Most common mutations associated with later phenotypes including p.I1061T, p.P1007A, and p.G992W result in changes in the cysteine-rich loop which has an important role in the cholesterol transport chain [85].

The following mutations so far have only been reported to only cause EIF in homozygous state: gross deletion of exon 1+promoter, c.3578_3591 + 9del, c.385delT, p.C63fsX75, IVS21-2delATGC, c. 2740T>A (p.C914S), c.3584G > T (p.G1195V), c.3478-6T>A, c.960_961dup (p.A321Gfs*16) (Table 3). IVS23+1G $>A$ is predicted to be a severe mutation, which causes EIF even in combination with p.A1035V or p.I1061T. c.1415T >C (p.L472P) and c.3394G $>$ C (p.A1132P) mutations were detected in both early and LIF patients in homozygous state. c.3107C > T (p.T1036M) and c.1553G >A (p.R518Q) often cause EIF, however, when in combination with late onset associated mutations such as c.3182C $>\mathrm{T}$ (p.I1061T), c.3019C > G (p.P1007A), and c.2861C $>$ T (p.S954L), they mostly present with a later onset phenotypes.

The NPC2 gene encodes a small lysosomal/late-endosomal glycoprotein that is ubiquitously expressed and plays a role in cholesterol trafficking $[8,9,86]$. Twenty-eight disease causing mutations have been reported so far (www.hgmd.cf.ac.uk) including 19 missense/nonsense mutations, four mutations affecting splicing, three small deletions, one small insertion/deletion, and one gross deletion. c.58G $>\mathrm{T}$ (p.E20X) and c.352G $>\mathrm{T}$ (p.E118X) were the most common mutations associated with EIF, however they were also reported in non-neurological phenotype with significant pulmonary involvement and visceromegaly. Whilst the patients did not appear to show obvious neurological features, it is likely that the early death pre-empted symptom evolution. c.434T $>$ A (p.V145E), c.436C $>\mathrm{T}$ (p.Q146X), c.199T>C (p.S67P), c.133C > T (p.Q45X), c.141C >A (p.C47X), and c.82+2T>C (IVS1+2T>C) mutations were also reported in EIF patients in a homozygous state. c.434T $>$ A (p.V145E), c.199T $>C$ (p.S67P), c.133C > T (p.Q45X), c.141C >A (p.C47X) mutations were only associated with EIF phenotype (Table 5). There was only one patient with compound heterozygous c.3G $>C$ (p.M1I) and c.190+5G $>A$ (IVS2+5G $>$ A) mutations. c.190+5G $>$ A (IVS2+5G $>$ A) was reported in a juvenile phenotype associated mutation [54,64]. c.3G $>$ C (p.M1I) is predicted to be a severe mutation which led to an EIF even in combination with a juvenile phenotype associated mutation.

There is a dearth of effective treatments available for NPC. Management is mainly symptomatic to increase the quality of life $[1,12]$. Patients with dysphagia should be closely monitored to avoid serious lung infections secondary to aspiration and to ensure adequate nutrition. Some patients need gastrostomy to maintain daily caloric intake $[18,84]$. Antiepileptic drugs are used although typically seizures are difficult to control [17]. Although tricyclic antidepressants have been used historically for cataplexy, their effect is very limited [17]. Dystonia and tremors may respond to anticholinergic drugs 
at least transiently, in some patients. Other drugs used for tone management include trihexyphenydil, benzodiazepines, botulinum toxin, and gamma-aminobutyric acid [17]. Physiotherapy can be used to delay the onset of contractures.

Miglustat is the only disease specific therapy approved for treatment of NPC worldwide. Miglustat is an iminosugar that inhibits glycosphingolipid synthesis. It has been reported to slow neurological deterioration and improve survival in different NPC cohorts $[42,84,87,88]$. Recently, a large retrospective observational study that used a multinational registry and five large national cohorts suggested that miglustat effect was statistically significant in improving the survival of LIF and juvenile patients, but not those in EIF group [89]. We could not obtain sufficient data on the miglustat use in the EIF group reviewed here to comment on its effect.

A number of experimental therapy trials are currently at different stages of clinical development. Arimoclomol upregulates molecular chaperones in cells including Hsp70, which increases the expression of mutant protein forms that may retain some of the function. The initial results of an Orphazyme-sponsored prospective, randomized, double-blind, placebo-controlled study suggested potential efficacy (NCT02612129) [90]. Hydroxypropyl-beta-cyclodextrin (HP $\beta C D$ ) is a molecule, which was shown to reduce neuronal cholesterol and ganglioside storage, decrease Purkinje cell death, and increase lifespan in many animal model studies [91-94]. A phase I/IIa trial of intrathecal HP $\beta C D$ demonstrated a slower neurological disease progression in treated patients (NCT02534844, NCT01747135). The clinical trials investigating intravenous (sponsored by CTD Holdings) and intrathecal (sponsored by Malinckrodt) HP $\beta C D$, as well as the combined administration (see clinicaltrials.govc) are ongoing [95,96]. Acetyl-DL-leucine, an acetylated derivative of a natural amino acid, significantly improved ataxic symptoms without side effects and quality of life $[97,98]$. A phase 2 clinical trial investigating the efficacy of Acetyl-L-Leucine sponsored by IntraBio is currently in progress (NCT03759639). Unfortunately, most of the clinical trials initiated so far excluded patients with early forms of the disease.

There has been a suggestion that bone marrow transplantation (BMT) may be effective in NPC2 as NPC2 is a soluble and secreted protein. However, only one NPC2 case treated with BMT and a long-term follow up could be found in the literature. A LIF NPC2 patient, presented with HSM and respiratory symptoms from the neonatal period including tachypnoea, recurrent infections, and oxygen dependence underwent BMT at the age of 16 months. Gradual improvement in respiratory symptoms and hepatosplenomegaly was reported by the age of 3 years. The child started to walk independently at the age of 24 months. However, after the age of 2 years the patient started regressing and at 33 months he no longer had any words. At 46 months of age, there was a decrease in his socialization with a reduction in vocalization. At the age of 63 months seizures started and became gradually intractable $[99,100]$.

Gene therapy is a rapidly evolving field and a number of pre-clinical studies have shown that it may hold potential in treating NPC [101-103]. These studies have involved adeno-associated viral vectors to deliver a functional copy of the NPC1 gene to a mouse model of NPC and reported neurological and systemic improvements and increased survival.

\section{Materials and Methods}

This review was based on a search through MEDLINE database using PubMed as the search engine. All relevant articles, original articles, case reports, and reviews published through April 2020 were included. The database was searched using the following medical subject headings (MeSH terms) "Niemann Pick Disease type C" and "Niemann Pick Disease". Furthermore, the references of the articles were investigated by hand for related articles. Our main focus were patients with EIF phenotype, therefore only cases with neurological symptoms in the first 2 years of life were included. Cases with late infantile (LIF), juvenile, and adult phenotypes were excluded. Cases presented with acute liver failure and treated with liver transplantation were not included in order to ensure uniformity. To evaluate the characteristics of EIF neurological disease, cases with "developmental delay" reported 
as the only symptom were also excluded. Only cases with at least one identified mutation in NPC1 or NPC2 were selected. In cases where only one mutation was identified, the diagnosis had to be supported by a biomarker test such as filipin staining of cultured skin fibroblasts. Other exclusion criteria used were (1) the article does not have abstract or the abstract is not available from the included electronic database; (2) the full text of the article is not available in English.

\section{Conclusions}

These data derived from an extensive review of NPC literature focused on the EIF type of NPC and provides an overview of the disease, with particular emphasis on neurological course and genetic features. Identification of mutations determining the EIF will help predict the disease progression and structure future clinical trials of novel therapies. Inevitably, only prospective natural history studies with set inclusion and exclusion criteria as well as detailed assessment categories could provide the granularity for the time course of the disease. This can only be achieved by close interaction of many international centers and family organizations.

Author Contributions: B.S.Y. and P.G. conceived the idea. B.S.Y. wrote the manuscript. J.B., A.A.R. and P.G. edited the manuscript. All authors have read and agreed to the published version of the manuscript.

Funding: B.S.Y. is supported by The Scientific and Technological Research Council of Turkey (TUBITAK). J.B. is in receipt of a Medical Research Council Fellowship MR/T008024/1. J.B. and P.G. are receiving support from the NIHR Great Ormond Street Hospital Biomedical Research Centre. The views expressed are those of the authors and not necessarily those of the NHS, the NIHR, or the Department of Health. A.A.R. is supported by the UK Medical Research Council (MR/N026101/1, MR/S036784/1, MR/R025134/1, MR/R015325/1, MR/S009434/1), the Wellcome Trust Institutional Strategic Support Fund/UCL Therapeutic Acceleration Support (TAS) Fund (204841/Z/16/Z), Action Medical Research (GN2485), and Asociación Niemann Pick de Fuenlabrada.

Conflicts of Interest: B.S.Y., J.B. and A.A.R has no conflict of interest. P.G. is an investigator on Malinckrodt, IntraBio, and Orphazyme sponsored clinical trials and received speaker and advisory board honoraria.

\section{References}

1. Vanier, M.T. Niemann-Pick disease type C. Orphanet J. Rare Dis. 2010, 5, 16. [CrossRef] [PubMed]

2. Patterson, M.C.; Mengel, E.; Wijburg, F.A.; Muller, A.; Schwierin, B.; Drevon, H.; Vanier, M.T.; Pineda, M. Disease and patient characteristics in NP-C patients: Findings from an international disease registry. Orphanet J. Rare Dis. 2013, 8, 12. [CrossRef] [PubMed]

3. Vanier, M.T.; Gissen, P.; Bauer, P.; Coll, M.J.; Burlina, A.; Hendriksz, C.J.; Latour, P.; Goizet, C.; Welford, R.W.; Marquardt, T.; et al. Diagnostic tests for Niemann-Pick disease type C (NP-C): A critical review. Mol. Genet. Metab. 2016, 118, 244-254. [CrossRef] [PubMed]

4. Hammond, N.; Munkacsi, A.B.; Sturley, S.L. The complexity of a monogenic neurodegenerative disease: More than two decades of therapeutic driven research into Niemann-Pick type C disease. Biochim. Biophys. Acta Mol. Cell. Biol. Lipids 2019, 1864, 1109-1123. [CrossRef] [PubMed]

5. Kwon, H.J.; Abi-Mosleh, L.; Wang, M.L.; Deisenhofer, J.; Goldstein, J.L.; Brown, M.S.; Infante, R.E. Structure of N-terminal domain of NPC1 reveals distinct subdomains for binding and transfer of cholesterol. Cell 2009, 137, 1213-1224. [CrossRef] [PubMed]

6. Infante, R.E.; Radhakrishnan, A.; Abi-Mosleh, L.; Kinch, L.N.; Wang, M.L.; Grishin, N.V.; Goldstein, J.L.; Brown, M.S. Purified NPC1 protein: II. Localization of sterol binding to a 240 -amino acid soluble luminal loop. J. Biol. Chem. 2008, 283, 1064-1075. [CrossRef]

7. Ioannou, Y.A. The structure and function of the Niemann-Pick C1 protein. Mol. Genet. Metab. 2000, 71, 175-181. [CrossRef]

8. Ko, D.C.; Binkley, J.; Sidow, A.; Scott, M.P. The integrity of a cholesterol-binding pocket in Niemann-Pick C2 protein is necessary to control lysosome cholesterol levels. Proc. Natl. Acad. Sci. USA 2003, 100, 2518-2525. [CrossRef]

9. Friedland, N.; Liou, H.L.; Lobel, P.; Stock, A.M. Structure of a cholesterol-binding protein deficient in Niemann-Pick type C2 disease. Proc. Natl. Acad. Sci. USA 2003, 100, 2512-2517. [CrossRef] 
10. Infante, R.E.; Wang, M.L.; Radhakrishnan, A.; Kwon, H.J.; Brown, M.S.; Goldstein, J.L. NPC2 facilitates bidirectional transfer of cholesterol between NPC1 and lipid bilayers, a step in cholesterol egress from lysosomes. Proc. Natl. Acad. Sci. USA 2008, 105, 15287-15292. [CrossRef]

11. Li, X.; Saha, P.; Li, J.; Blobel, G.; Pfeffer, S.R. Clues to the mechanism of cholesterol transfer from the structure of NPC1 middle lumenal domain bound to NPC2. Proc. Natl. Acad. Sci. USA 2016, 113, 10079-10084. [CrossRef] [PubMed]

12. Geberhiwot, T.; Moro, A.; Dardis, A.; Ramaswami, U.; Sirrs, S.; Marfa, M.P.; Vanier, M.T.; Walterfang, M.; Bolton, S.; Dawson, C.; et al. Consensus clinical management guidelines for Niemann-Pick disease type C. Orphanet J. Rare Dis. 2018, 13, 50. [CrossRef] [PubMed]

13. Mavridou, I.; Cozar, M.; Douzgou, S.; Xaidara, A.; Lianou, D.; Vanier, M.T.; Dimitriou, E.; Grinberg, D.; Vilageliu, L.; Michelakakis, H. Niemann-Pick type C disease: A novel NPC1 mutation segregating in a Greek island. Clin. Genet. 2014, 85, 543-547. [CrossRef] [PubMed]

14. Millat, G.; Marcais, C.; Rafi, M.A.; Yamamoto, T.; Morris, J.A.; Pentchev, P.G.; Ohno, K.; Wenger, D.A.; Vanier, M.T. Niemann-Pick C1 disease: The I1061T substitution is a frequent mutant allele in patients of Western European descent and correlates with a classic juvenile phenotype. Am. J. Hum. Genet. 1999, 65, 1321-1329. [CrossRef]

15. McKay Bounford, K.; Gissen, P. Genetic and laboratory diagnostic approach in Niemann Pick disease type C. J. Neurol. 2014, 261, S569-S575. [CrossRef]

16. Wassif, C.A.; Cross, J.L.; Iben, J.; Sanchez-Pulido, L.; Cougnoux, A.; Platt, F.M.; Ory, D.S.; Ponting, C.P.; Bailey-Wilson, J.E.; Biesecker, L.G.; et al. High incidence of unrecognized visceral/neurological late-onset Niemann-Pick disease, type C1, predicted by analysis of massively parallel sequencing data sets. Genet. Med. 2016, 18, 41-48. [CrossRef]

17. Patterson, M.C.; Hendriksz, C.J.; Walterfang, M.; Sedel, F.; Vanier, M.T.; Wijburg, F.; Group, N.-C.G.W. Recommendations for the diagnosis and management of Niemann-Pick disease type C: An update. Mol. Genet. Metab. 2012, 106, 330-344. [CrossRef]

18. Wraith, J.E.; Guffon, N.; Rohrbach, M.; Hwu, W.L.; Korenke, G.C.; Bembi, B.; Luzy, C.; Giorgino, R.; Sedel, F. Natural history of Niemann-Pick disease type $\mathrm{C}$ in a multicentre observational retrospective cohort study. Mol. Genet. Metab. 2009, 98, 250-254. [CrossRef]

19. Imrie, J.; Dasgupta, S.; Besley, G.T.; Harris, C.; Heptinstall, L.; Knight, S.; Vanier, M.T.; Fensom, A.H.; Ward, C.; Jacklin, E.; et al. The natural history of Niemann-Pick disease type C in the UK. J. Inherit. Metab. Dis. 2007, 30, 51-59. [CrossRef]

20. Garver, W.S.; Francis, G.A.; Jelinek, D.; Shepherd, G.; Flynn, J.; Castro, G.; Walsh Vockley, C.; Coppock, D.L.; Pettit, K.M.; Heidenreich, R.A.; et al. The National Niemann-Pick C1 disease database: Report of clinical features and health problems. Am. J. Med. Genet. A 2007, 143A, 1204-1211. [CrossRef]

21. Stampfer, M.; Theiss, S.; Amraoui, Y.; Jiang, X.; Keller, S.; Ory, D.S.; Mengel, E.; Fischer, C.; Runz, H. Niemann-Pick disease type $C$ clinical database: Cognitive and coordination deficits are early disease indicators. Orphanet J. Rare Dis. 2013, 8, 35. [CrossRef] [PubMed]

22. Lipinski, P.; Jankowska, I.; Lugowska, A.; Musielak, M.; Pronicki, M.; Tylki-Szymanska, A. Newborn presentation of Niemann-Pick disease type C-Difficulties and limitations of diagnostic methods. Pediatr. Neonatol. 2018, 59, 317-318. [CrossRef] [PubMed]

23. Kumagai, T.; Terashima, H.; Uchida, H.; Fukuda, A.; Kasahara, M.; Kosuga, M.; Okuyama, T.; Tsunoda, T.; Inui, A.; Fujisawa, T.; et al. A case of Niemann-Pick disease type $C$ with neonatal liver failure initially diagnosed as neonatal hemochromatosis. Brain Dev. 2019, 41, 460-464. [CrossRef] [PubMed]

24. Kelly, D.A.; Portmann, B.; Mowat, A.P.; Sherlock, S.; Lake, B.D. Niemann-Pick disease type C: Diagnosis and outcome in children, with particular reference to liver disease. J. Pediatr. 1993, 123, 242-247. [CrossRef]

25. Yamada, N.; Inui, A.; Sanada, Y.; Ihara, Y.; Urahashi, T.; Fukuda, A.; Sakamoto, S.; Kasahara, M.; Yoshizawa, A.; Okamoto, S.; et al. Pediatric liver transplantation for neonatal-onset Niemann-Pick disease type C: Japanese multicenter experience. Pediatr. Transplant. 2019, 23, e13462. [CrossRef]

26. Hegarty, R.; Hadzic, N.; Gissen, P.; Dhawan, A. Inherited metabolic disorders presenting as acute liver failure in newborns and young children: King's College Hospital experience. Eur. J. Pediatr. 2015, 174, 1387-1392. [CrossRef] 
27. Spiegel, R.; Raas-Rothschild, A.; Reish, O.; Regev, M.; Meiner, V.; Bargal, R.; Sury, V.; Meir, K.; Nadjari, M.; Hermann, G.; et al. The clinical spectrum of fetal Niemann-Pick type C. Am. J. Med. Genet. A 2009, 149A, 446-450. [CrossRef]

28. Maconochie, I.K.; Chong, S.; Mieli-Vergani, G.; Lake, B.D.; Mowat, A.P. Fetal ascites: An unusual presentation of Niemann-Pick disease type C. Arch. Dis. Child. 1989, 64, 1391-1393. [CrossRef]

29. Surmeli-Onay, O.; Yakarisik, S.; Korkmaz, A.; Akcoren, Z.; Yuce, A.; Runz, H.; Stampfer, M.; Yurdakok, M. Prenatal-onset Niemann-Pick type C disease with nonimmune hydrops fetalis. Pediatr. Neonatol. 2013, 54, 344-347. [CrossRef]

30. Mengel, E.; Klunemann, H.H.; Lourenco, C.M.; Hendriksz, C.J.; Sedel, F.; Walterfang, M.; Kolb, S.A. Niemann-Pick disease type C symptomatology: An expert-based clinical description. Orphanet J. Rare. Dis. 2013, 8, 166. [CrossRef]

31. Yanjanin, N.M.; Velez, J.I.; Gropman, A.; King, K.; Bianconi, S.E.; Conley, S.K.; Brewer, C.C.; Solomon, B.; Pavan, W.J.; Arcos-Burgos, M.; et al. Linear clinical progression, independent of age of onset, in Niemann-Pick disease, type C. Am. J. Med. Genet. B Neuropsychiatr. Genet. 2010, 153B, 132-140. [CrossRef] [PubMed]

32. Walterfang, M.; Fietz, M.; Fahey, M.; Sullivan, D.; Leane, P.; Lubman, D.I.; Velakoulis, D. The neuropsychiatry of Niemann-Pick type C disease in adulthood. J. Neuropsychiatry Clin. Neurosci. 2006, 18, 158-170. [CrossRef]

33. Klarner, B.; Klunemann, H.H.; Lurding, R.; Aslanidis, C.; Rupprecht, R. Neuropsychological profile of adult patients with Niemann-Pick C1 (NPC1) mutations. J. Inherit. Metab. Dis. 2007, 30, 60-67. [CrossRef] [PubMed]

34. Patterson, M.C.; Clayton, P.; Gissen, P.; Anheim, M.; Bauer, P.; Bonnot, O.; Dardis, A.; Dionisi-Vici, C.; Klunemann, H.H.; Latour, P.; et al. Recommendations for the detection and diagnosis of Niemann-Pick disease type C: An update. Neurol. Clin. Pract. 2017, 7, 499-511. [CrossRef] [PubMed]

35. Rego, T.; Farrand, S.; Goh, A.M.Y.; Eratne, D.; Kelso, W.; Mangelsdorf, S.; Velakoulis, D.; Walterfang, M. Psychiatric and Cognitive Symptoms Associated with Niemann-Pick Type C Disease: Neurobiology and Management. CNS Drugs 2019, 33, 125-142. [CrossRef]

36. Jahnova, H.; Dvorakova, L.; Vlaskova, H.; Hulkova, H.; Poupetova, H.; Hrebicek, M.; Jesina, P. Observational, retrospective study of a large cohort of patients with Niemann-Pick disease type $C$ in the Czech Republic: A surprisingly stable diagnostic rate spanning almost 40 years. Orphanet J. Rare Dis. 2014, 9, 140. [CrossRef]

37. Imrie, J.; Heptinstall, L.; Knight, S.; Strong, K. Observational cohort study of the natural history of Niemann-Pick disease type C in the UK: A 5-year update from the UK clinical database. BMC Neurol. 2015, 15, 257. [CrossRef]

38. Fernandez-Valero, E.M.; Ballart, A.; Iturriaga, C.; Lluch, M.; Macias, J.; Vanier, M.T.; Pineda, M.; Coll, M.J. Identification of 25 new mutations in 40 unrelated Spanish Niemann-Pick type C patients: Genotype-phenotype correlations. Clin. Genet. 2005, 68, 245-254. [CrossRef]

39. Macias-Vidal, J.; Rodriguez-Pascau, L.; Sanchez-Olle, G.; Lluch, M.; Vilageliu, L.; Grinberg, D.; Coll, M.J.; Spanish, N.P.C.W.G. Molecular analysis of 30 Niemann-Pick type C patients from Spain. Clin. Genet. 2011, 80, 39-49. [CrossRef]

40. Fancello, T.; Dardis, A.; Rosano, C.; Tarugi, P.; Tappino, B.; Zampieri, S.; Pinotti, E.; Corsolini, F.; Fecarotta, S.; D'Amico, A.; et al. Molecular analysis of NPC1 and NPC2 gene in 34 Niemann-Pick C Italian patients: Identification and structural modeling of novel mutations. Neurogenetics 2009, 10, 229-239. [CrossRef]

41. Dardis, A.; Zampieri, S.; Gellera, C.; Carrozzo, R.; Cattarossi, S.; Peruzzo, P.; Dariol, R.; Sechi, A.; Deodato, F.; Caccia, C.; et al. Molecular Genetics of Niemann-Pick Type C Disease in Italy: An Update on 105 Patients and Description of 18 NPC1 Novel Variants. J. Clin. Med. 2020, 9, 679. [CrossRef] [PubMed]

42. Heron, B.; Valayannopoulos, V.; Baruteau, J.; Chabrol, B.; Ogier, H.; Latour, P.; Dobbelaere, D.; Eyer, D.; Labarthe, F.; Maurey, H.; et al. Miglustat therapy in the French cohort of paediatric patients with Niemann-Pick disease type C. Orphanet J. Rare Dis. 2012, 7, 36. [CrossRef]

43. Mahmoud, I.G.; Elmonem, M.A.; Elkhateeb, N.M.; Elnaggar, W.; Sobhi, A.; Girgis, M.Y.; Kamel, M.; Shaheen, Y.; Samaha, M.; Ramadan, A.; et al. Clinical, biomarker and genetic spectrum of Niemann-Pick type C in Egypt: The detection of nine novel NPC1 mutations. Clin. Genet. 2019, 95, 537-539. [CrossRef] [PubMed] 
44. Karimzadeh, P.; Tonekaboni, S.H.; Ashrafi, M.R.; Shafeghati, Y.; Rezayi, A.; Salehpour, S.; Ghofrani, M.; Taghdiri, M.M.; Rahmanifar, A.; Zaman, T.; et al. Effects of miglustat on stabilization of neurological disorder in niemann-pick disease type C: Iranian pediatric case series. J. Child. Neurol. 2013, 28, 1599-1606. [CrossRef] [PubMed]

45. Hashemian, S.; Eshraghi, P.; Dilaver, N.; Galehdari, H.; Shalbafan, B.; Vakili, R.; Ghaemi, N.; Ahangari, N.; Rezazadeh Varaghchi, J.; Zeighami, J.; et al. Niemann-Pick Diseases: The Largest Iranian Cohort with Genetic Analysis. Iran. J. Child. Neurol. 2019, 13, 155-162. [PubMed]

46. Zhang, H.; Wang, Y.; Lin, N.; Yang, R.; Qiu, W.; Han, L.; Ye, J.; Gu, X. Diagnosis of Niemann-Pick disease type $\mathrm{C}$ with 7-ketocholesterol screening followed by NPC1/NPC2 gene mutation confirmation in Chinese patients. Orphanet J. Rare. Dis. 2014, 9, 82. [CrossRef] [PubMed]

47. Staretz-Chacham, O.; Aviram, M.; Morag, I.; Goldbart, A.; Hershkovitz, E. Pulmonary involvement in Niemann-Pick C type 1. Eur. J. Pediatr. 2018, 177, 1609-1615. [CrossRef]

48. Lopez De Frutos, L.; Cebolla, J.J.; Aldamiz-Echevarria, L.; De La Vega, A.; Stanescu, S.; Lahoz, C.; Irun, P.; Giraldo, P. New variants in Spanish Niemann-Pick type c disease patients. Mol. Biol. Rep. 2020, 47, 2085-2095. [CrossRef]

49. Roch Santed, M.; Cabanas Poy, M.J.; Del Toro Riera, M.; Canete Ramirez, C.; Fernandez Polo, A.; Clemente Bautista, S. Intrathecal cyclodextrin in the treatment of Niemann-Pick disease type C. Eur. J. Hosp. Pharm. 2017, 24, 185-188. [CrossRef]

50. Kodachi, T.; Matsumoto, S.; Mizuguchi, M.; Osaka, H.; Kanai, N.; Nanba, E.; Ohno, K.; Yamagata, T. Severe demyelination in a patient with a late infantile form of Niemann-Pick disease type C. Neuropathology 2017, 37, 426-430. [CrossRef]

51. Usui, M.; Miyauchi, A.; Nakano, Y.; Nakamura, S.; Jimbo, E.; Itamura, S.; Adachi, K.; Nanba, E.; Narita, A.; Yamagata, T.; et al. Miglustat therapy in a case of early-infantile Niemann-Pick type C. Brain Dev. 2017, 39, 886-890. [CrossRef]

52. Ribeiro, I.; Marcao, A.; Amaral, O.; Sa Miranda, M.C.; Vanier, M.T.; Millat, G. Niemann-Pick type C disease: NPC1 mutations associated with severe and mild cellular cholesterol trafficking alterations. Hum. Genet. 2001, 109, 24-32. [CrossRef] [PubMed]

53. Noroozi Asl, S.; Vakili, R.; Ghaemi, N.; Eshraghi, P. The Report of Three Rare Cases of the Niemann-pick Disease in Birjand, South Khorasan, Eastern Iran. Iran. J. Child. Neurol. 2017, 11, 53-56. [PubMed]

54. Mavridou, I.; Dimitriou, E.; Vanier, M.T.; Vilageliu, L.; Grinberg, D.; Latour, P.; Xaidara, A.; Lycopoulou, L.; Bostantjopoulou, S.; Zafeiriou, D.; et al. The Spectrum of Niemann-Pick Type C Disease in Greece. J. Inh. Metab. Dis. Rep. 2017, 36, 41-48. [CrossRef]

55. Millat, G.; Marcais, C.; Tomasetto, C.; Chikh, K.; Fensom, A.H.; Harzer, K.; Wenger, D.A.; Ohno, K.; Vanier, M.T. Niemann-Pick C1 disease: Correlations between NPC1 mutations, levels of NPC1 protein, and phenotypes emphasize the functional significance of the putative sterol-sensing domain and of the cysteine-rich luminal loop. Am. J. Hum. Genet. 2001, 68, 1373-1385. [CrossRef] [PubMed]

56. Vanier, M.T.; Wenger, D.A.; Comly, M.E.; Rousson, R.; Brady, R.O.; Pentchev, P.G. Niemann-Pick disease group C: Clinical variability and diagnosis based on defective cholesterol esterification. A collaborative study on 70 patients. Clin. Genet. 1988, 33, 331-348. [CrossRef]

57. Rodriguez-Pascau, L.; Toma, C.; Macias-Vidal, J.; Cozar, M.; Cormand, B.; Lykopoulou, L.; Coll, M.J.; Grinberg, D.; Vilageliu, L. Characterisation of two deletions involving NPC1 and flanking genes in Niemann-Pick type C disease patients. Mol. Genet. Metab. 2012, 107, 716-720. [CrossRef]

58. Pedroso, J.L.; Fusao, E.F.; Ladeia-Frota, C.; Arita, J.H.; Barsottini, O.G.; Masruha, M.R.; Vilanova, L.C. Teaching video neuroimages: Gelastic cataplexy as the first neurologic manifestation of Niemann-Pick disease type C. Neurology 2012, 79, e189. [CrossRef]

59. Degtyareva, A.V.; Proshlyakova, T.Y.; Gautier, M.S.; Degtyarev, D.N.; Kamenets, E.A.; Baydakova, G.V.; Rebrikov, D.V.; Zakharova, E.Y. Oxysterol/chitotriosidase based selective screening for Niemann-Pick type C in infantile cholestasis syndrome patients. BMC Med. Genet. 2019, 20, 123. [CrossRef]

60. Xiong, H.; Higaki, K.; Wei, C.J.; Bao, X.H.; Zhang, Y.H.; Fu, N.; Qin, J.; Adachi, K.; Kumura, Y.; Ninomiya, H.; et al. Genotype/phenotype of 6 Chinese cases with Niemann-Pick disease type C. Gene 2012, 498, 332-335. [CrossRef] 
61. Takamura, A.; Sakai, N.; Shinpoo, M.; Noguchi, A.; Takahashi, T.; Matsuda, S.; Yamamoto, M.; Narita, A.; Ohno, K.; Ohashi, T.; et al. The useful preliminary diagnosis of Niemann-Pick disease type C by filipin test in blood smear. Mol. Genet. Metab. 2013, 110, 401-404. [CrossRef] [PubMed]

62. Yamamoto, T.; Nanba, E.; Ninomiya, H.; Higaki, K.; Taniguchi, M.; Zhang, H.; Akaboshi, S.; Watanabe, Y.; Takeshima, T.; Inui, K.; et al. NPC1 gene mutations in Japanese patients with Niemann-Pick disease type C. Hum. Genet. 1999, 105, 10-16. [CrossRef]

63. Yamamoto, T.; Ninomiya, H.; Matsumoto, M.; Ohta, Y.; Nanba, E.; Tsutsumi, Y.; Yamakawa, K.; Millat, G.; Vanier, M.T.; Pentchev, P.G.; et al. Genotype-phenotype relationship of Niemann-Pick disease type C: A possible correlation between clinical onset and levels of NPC1 protein in isolated skin fibroblasts. J. Med. Genet. 2000, 37, 707-712. [CrossRef] [PubMed]

64. Millat, G.; Chikh, K.; Naureckiene, S.; Sleat, D.E.; Fensom, A.H.; Higaki, K.; Elleder, M.; Lobel, P.; Vanier, M.T. Niemann-Pick disease type C: Spectrum of HE1 mutations and genotype/phenotype correlations in the NPC2 group. Am. J. Hum. Genet. 2001, 69, 1013-1021. [CrossRef] [PubMed]

65. Reunert, J.; Lotz-Havla, A.S.; Polo, G.; Kannenberg, F.; Fobker, M.; Griese, M.; Mengel, E.; Muntau, A.C.; Schnabel, P.; Sommerburg, O.; et al. Niemann-Pick Type C-2 Disease: Identification by Analysis of Plasma Cholestane-3beta,5alpha,6beta-Triol and Further Insight into the Clinical Phenotype. JIMD Rep. 2015, 23, 17-26. [CrossRef]

66. Verot, L.; Chikh, K.; Freydiere, E.; Honore, R.; Vanier, M.T.; Millat, G. Niemann-Pick C disease: Functional characterization of three NPC2 mutations and clinical and molecular update on patients with NPC2. Clin. Genet. 2007, 71, 320-330. [CrossRef]

67. Topcu, M.; Aktas, D.; Oztoprak, M.; Mungan, N.O.; Yuce, A.; Alikasifoglu, M. Prospective Turkish Cohort Study to Investigate the Frequency of Niemann-Pick Disease Type C Mutations in Consanguineous Families with at Least One Homozygous Family Member. Mol. Diagn. Ther. 2017, 21, 643-651. [CrossRef]

68. Yaman, A.; Eminoglu, F.T.; Kendirli, T.; Odek, C.; Ceylaner, S.; Kansu, A.; Ince, E.; Deda, G. A rare cause of fatal pulmonary alveolar proteinosis: Niemann-Pick disease type $\mathrm{C} 2$ and a novel mutation. J. Pediatr. Endocrinol. Metab. 2015, 28, 1163-1167. [CrossRef]

69. Vanier, M.T.; Duthel, S.; Rodriguez-Lafrasse, C.; Pentchev, P.; Carstea, E.D. Genetic heterogeneity in Niemann-Pick C disease: A study using somatic cell hybridization and linkage analysis. Am. J. Hum. Genet. 1996, 58, 118-125.

70. Chikh, K.; Rodriguez, C.; Vey, S.; Vanier, M.T.; Millat, G. Niemann-Pick type C disease: Subcellular location and functional characterization of NPC2 proteins with naturally occurring missense mutations. Hum. Mutat. 2005, 26, 20-28. [CrossRef]

71. Sheth, J.; Joseph, J.J.; Shah, K.; Muranjan, M.; Mistri, M.; Sheth, F. Pulmonary manifestations in Niemann-Pick type C disease with mutations in NPC2 gene: Case report and review of literature. BMC Med. Genet. 2017, 18, 5. [CrossRef]

72. Millat, G.; Bailo, N.; Molinero, S.; Rodriguez, C.; Chikh, K.; . Vanier, M.T. Niemann-Pick C disease: Use of denaturing high performance liquid chromatography for the detection of NPC1 and NPC2 genetic variations and impact on management of patients and families. Mol. Genet. Metab. 2005, 86, 220-232. [CrossRef] [PubMed]

73. Wijburg, F.A.; Sedel, F.; Pineda, M.; Hendriksz, C.J.; Fahey, M.; Walterfang, M.; Patterson, M.C.; Wraith, J.E.; Kolb, S.A. Development of a suspicion index to aid diagnosis of Niemann-Pick disease type C. Neurology 2012, 78, 1560-1567. [CrossRef] [PubMed]

74. Bjurulf, B.; Spetalen, S.; Erichsen, A.; Vanier, M.T.; Strom, E.H.; Stromme, P. Niemann-Pick disease type C2 presenting as fatal pulmonary alveolar lipoproteinosis: Morphological findings in lung and nervous tissue. Med. Sci. Monit. 2008, 14, CCS71-CCS75. [PubMed]

75. Elleder, M.; Houstkova, H.; Zeman, J.; Ledvinova, J.; Poupetova, H. Pulmonary storage with emphysema as a sign of Niemann-Pick type C2 disease (second complementation group). Report of a case. Virchows Arch. 2001, 439, 206-211. [CrossRef] [PubMed]

76. Griese, M.; Brasch, F.; Aldana, V.R.; Cabrera, M.M.; Goelnitz, U.; Ikonen, E.; Karam, B.J.; Liebisch, G.; Linder, M.D.; Lohse, P.; et al. Respiratory disease in Niemann-Pick type C2 is caused by pulmonary alveolar proteinosis. Clin. Genet. 2010, 77, 119-130. [CrossRef]

77. Guillemot, N.; Troadec, C.; De Villemeur, T.B.; Clement, A.; Fauroux, B. Lung disease in Niemann-Pick disease. Pediatr. Pulmonol. 2007, 42, 1207-1214. [CrossRef] 
78. Morisot, C.; Millat, G.; Coeslier, A.; Bourgois, B.; Fontenoy, E.; Dobbelaere, D.; Verot, L.; Haouari, N.; Vaillant, C.; Gottrand, F.; et al. [Fatal neonatal respiratory distress in Niemann-Pick C2 and prenatal diagnosis with mutations in gene HE1/NPC2]. Arch. Pediatr. 2005, 12, 434-437. [CrossRef]

79. Schofer, O.; Mischo, B.; Puschel, W.; Harzer, K.; Vanier, M.T. Early-lethal pulmonary form of Niemann-Pick type $C$ disease belonging to a second, rare genetic complementation group. Eur. J. Pediatr. 1998, 157, 45-49. [CrossRef]

80. Palmeri, S.; Tarugi, P.; Sicurelli, F.; Buccoliero, R.; Malandrini, A.; De Santi, M.M.; Marciano, G.; Battisti, C.; Dotti, M.T.; Calandra, S.; et al. Lung involvement in Niemann-Pick disease type C1: Improvement with bronchoalveolar lavage. Neurol. Sci. 2005, 26, 171-173. [CrossRef]

81. Pineda, M.; Mengel, E.; Jahnova, H.; Heron, B.; Imrie, J.; Lourenco, C.M.; Van Der Linden, V.; Karimzadeh, P.; Valayannopoulos, V.; Jesina, P.; et al. A Suspicion Index to aid screening of early-onset Niemann-Pick disease Type C (NP-C). BMC Pediatr. 2016, 16, 107. [CrossRef] [PubMed]

82. Gburek-Augustat, J.; Groeschel, S.; Kern, J.; Beck-Woedl, S.; Just, J.; Harzer, K.; Stampfer, M.; Kraegeloh-Mann, I. Comparative Analysis of Cerebral Magnetic Resonance Imaging Changes in Nontreated Infantile, Juvenile and Adult Patients with Niemann-Pick Disease Type, C. Neuropediatrics 2020, 51, 37-44. [CrossRef] [PubMed]

83. Pineda, M.; Perez-Poyato, M.S.; O'Callaghan, M.; Vilaseca, M.A.; Pocovi, M.; Domingo, R.; Portal, L.R.; Perez, A.V.; Temudo, T.; Gaspar, A.; et al. Clinical experience with miglustat therapy in pediatric patients with Niemann-Pick disease type C: A case series. Mol. Genet. Metab. 2010, 99, 358-366. [CrossRef] [PubMed]

84. Walterfang, M.; Chien, Y.H.; Imrie, J.; Rushton, D.; Schubiger, D.; Patterson, M.C. Dysphagia as a risk factor for mortality in Niemann-Pick disease type C: Systematic literature review and evidence from studies with miglustat. Orphanet J. Rare Dis. 2012, 7, 76. [CrossRef]

85. Martinez-Archundia, M.; Hernandez Mojica, T.G.; Correa-Basurto, J.; Montano, S.; Camacho-Molina, A. Molecular dynamics simulations reveal structural differences among wild-type NPC1 protein and its mutant forms. J. Biomol. Struct. Dyn. 2019, 1-6. [CrossRef]

86. Naureckiene, S.; Sleat, D.E.; Lackland, H.; Fensom, A.; Vanier, M.T.; Wattiaux, R.; Jadot, M.; Lobel, P. Identification of HE1 as the second gene of Niemann-Pick C disease. Science 2000, 290, 2298-2301. [CrossRef]

87. Nadjar, Y.; Hutter-Moncada, A.L.; Latour, P.; Ayrignac, X.; Kaphan, E.; Tranchant, C.; Cintas, P.; Degardin, A.; Goizet, C.; Laurencin, C.; et al. Adult Niemann-Pick disease type C in France: Clinical phenotypes and long-term miglustat treatment effect. Orphanet J. Rare Dis. 2018, 13, 175. [CrossRef]

88. Patterson, M.C.; Mengel, E.; Vanier, M.T.; Schwierin, B.; Muller, A.; Cornelisse, P.; Pineda, M. Investigators, N.P.C.R. Stable or improved neurological manifestations during miglustat therapy in patients from the international disease registry for Niemann-Pick disease type C: An observational cohort study. Orphanet J. Rare Dis. 2015, 10, 65. [CrossRef]

89. Patterson, M.C.; Garver, W.S.; Giugliani, R.; Imrie, J.; Jahnova, H.; Meaney, F.J.; Nadjar, Y.; Vanier, M.T.; Moneuse, P.; Morand, O.; et al. Long-term survival outcomes of patients with Niemann-Pick disease type C receiving miglustat treatment: A large retrospective observational study. J. Inherit. Metab. Dis. 2020. [CrossRef]

90. Kirkegaard, T.; Gray, J.; Priestman, D.A.; Wallom, K.L.; Atkins, J.; Olsen, O.D.; Klein, A.; Drndarski, S.; Petersen, N.H.; Ingemann, L.; et al. Heat shock protein-based therapy as a potential candidate for treating the sphingolipidoses. Sci. Transl. Med. 2016, 8, 355ra118. [CrossRef]

91. Camargo, F.; Erickson, R.P.; Garver, W.S.; Hossain, G.S.; Carbone, P.N.; Heidenreich, R.A.; Blanchard, J. Cyclodextrins in the treatment of a mouse model of Niemann-Pick C disease. Life Sci. 2001, 70, 131-142. [CrossRef]

92. Liu, B.; Turley, S.D.; Burns, D.K.; Miller, A.M.; Repa, J.J.; Dietschy, J.M. Reversal of defective lysosomal transport in NPC disease ameliorates liver dysfunction and neurodegeneration in the npc1-/- mouse. Proc. Natl. Acad. Sci. USA 2009, 106, 2377-2382. [CrossRef] [PubMed]

93. Davidson, C.D.; Ali, N.F.; Micsenyi, M.C.; Stephney, G.; Renault, S.; Dobrenis, K.; Ory, D.S.; Vanier, M.T.; Walkley, S.U. Chronic cyclodextrin treatment of murine Niemann-Pick C disease ameliorates neuronal cholesterol and glycosphingolipid storage and disease progression. PLoS ONE 2009, 4, e6951. [CrossRef] [PubMed] 
94. Vite, C.H.; Bagel, J.H.; Swain, G.P.; Prociuk, M.; Sikora, T.U.; Stein, V.M.; O’Donnell, P.; Ruane, T.; Ward, S.; Crooks, A.; et al. Intracisternal cyclodextrin prevents cerebellar dysfunction and Purkinje cell death in feline Niemann-Pick type C1 disease. Sci. Transl. Med. 2015, 7, 276ra226. [CrossRef]

95. Farmer, C.A.; Thurm, A.; Farhat, N.; Bianconi, S.; Keener, L.A.; Porter, F.D. Long-Term Neuropsychological Outcomes from an Open-Label Phase I/IIa Trial of 2-Hydroxypropyl-beta-Cyclodextrins (VTS-270) in Niemann-Pick Disease, Type C1. CNS Drugs 2019, 33, 677-683. [CrossRef] [PubMed]

96. Ory, D.S.; Ottinger, E.A.; Farhat, N.Y.; King, K.A.; Jiang, X.; Weissfeld, L.; Berry-Kravis, E.; Davidson, C.D.; Bianconi, S.; Keener, L.A.; et al. Intrathecal 2-hydroxypropyl-beta-cyclodextrin decreases neurological disease progression in Niemann-Pick disease, type C1: A non-randomised, open-label, phase 1-2 trial. Lancet 2017, 390, 1758-1768. [CrossRef]

97. Strupp, M.; Teufel, J.; Habs, M.; Feuerecker, R.; Muth, C.; Van De Warrenburg, B.P.; Klopstock, T.; Feil, K. Effects of acetyl-DL-leucine in patients with cerebellar ataxia: A case series. J. Neurol. 2013, 260, 2556-2561. [CrossRef]

98. Bremova, T.; Malinova, V.; Amraoui, Y.; Mengel, E.; Reinke, J.; Kolnikova, M.; Strupp, M. Acetyl-dl-leucine in Niemann-Pick type C: A case series. Neurology 2015, 85, 1368-1375. [CrossRef]

99. Breen, C.; Wynn, R.F.; O’Meara, A.; O'Mahony, E.; Rust, S.; Imrie, J.; Wraith, J.E. Developmental outcome post allogenic bone marrow transplant for Niemann Pick Type C2. Mol. Genet. Metab. 2013, 108, 82-84. [CrossRef]

100. Bonney, D.K.; O’Meara, A.; Shabani, A.; Imrie, J.; Bigger, B.W.; Jones, S.; Wraith, J.E.; Wynn, R.F. Successful allogeneic bone marrow transplant for Niemann-Pick disease type $\mathrm{C} 2$ is likely to be associated with a severe 'graft versus substrate' effect. J. Inherit. Metab. Dis. 2010, 33, S171-S173. [CrossRef]

101. Hughes, M.P.; Smith, D.A.; Morris, L.; Fletcher, C.; Colaco, A.; Huebecker, M.; Tordo, J.; Palomar, N.; Massaro, G.; Henckaerts, E.; et al. AAV9 intracerebroventricular gene therapy improves lifespan, locomotor function and pathology in a mouse model of Niemann-Pick type C1 disease. Hum. Mol. Genet. 2018, 27, 3079-3098. [CrossRef] [PubMed]

102. Chandler, R.J.; Williams, I.M.; Gibson, A.L.; Davidson, C.D.; Incao, A.A.; Hubbard, B.T.; Porter, F.D.; Pavan, W.J.; Venditti, C.P. Systemic AAV9 gene therapy improves the lifespan of mice with Niemann-Pick disease, type C1. Hum. Mol. Genet. 2017, 26, 52-64. [CrossRef] [PubMed]

103. Xie, C.; Gong, X.M.; Luo, J.; Li, B.L.; Song, B.L. AAV9-NPC1 significantly ameliorates Purkinje cell death and behavioral abnormalities in mouse NPC disease. J. Lipid Res. 2017, 58, 512-518. [CrossRef] [PubMed] 